Coco, A, Currenti, G, Del Negro, C and Russo, G

A Second Order Finite-Difference Ghost-Point Method for Elasticity Problems on Unbounded Domains with Applications to Volcanology.

Coco, A, Currenti, G, Del Negro, C and Russo, G (2014) A Second Order Finite-Difference Ghost-Point Method for Elasticity Problems on Unbounded Domains with Applications to Volcanology. Communications in Computational Physics, 16 (04). pp. 983-1009.

doi: 10.4208/cicp.210713.010414a

This version is available: https://radar.brookes.ac.uk/radar/items/47a2ebd8-4aa9-415f-b8b9-c922764edd09/1/

Available on RADAR: July 2016

Copyright (C) and Moral Rights are retained by the author(s) and/ or other copyright owners. A copy can be downloaded for personal non-commercial research or study, without prior permission or charge. This item cannot be reproduced or quoted extensively from without first obtaining permission in writing from the copyright holder(s). The content must not be changed in any way or sold commercially in any format or medium without the formal permission of the copyright holders.

This document is the post print version of the journal article. Some differences between the published version and this version may remain and you are advised to consult the published version if you wish to cite from it. 


\title{
A Second Order Finite-Difference Ghost-Point Method for Elasticity Problems on unbounded domains with applications to Volcanology
}

\author{
Armando Coco $^{1, *}$, Gilda Currenti ${ }^{2}$, Ciro Del Negro ${ }^{2}$, and Gio- \\ vanni Russo ${ }^{3}$ \\ ${ }^{1}$ Dipartimento di Scienze della Terra e Geoambientali, Università di Bari Aldo Moro, \\ Bari, Italy. \\ 2 Istituto Nazionale di Geofisica e Vulcanologia \\ ${ }^{3}$ Dipartimento di Matematica e Informatica, Università di Catania, Catania, Italy
}

\begin{abstract}
We propose a finite-difference ghost-point approach for the numerical solution of Cauchy-Navier equations in linear elasticity problems on arbitrary unbounded domains. The technique is based on a smooth coordinate transformation, which maps an unbounded domain into a unit square. Arbitrary geometries are defined by suitable level-set functions. The equations are discretized by classical nine-point stencil on interior points, while boundary conditions and high order reconstructions are used to define the field variable at ghost-point, which are grid nodes external to the domain with a neighbor inside the domain. The linear system arising from such discretization is solved by a multigrid strategy. The approach is then applied to solve elasticity problems in volcanology for computing the displacement caused by pressure sources. The method is suitable to treat problems in which the geometry of the source often changes (explore the effects of different scenarios, or solve inverse problems in which the geometry itself is part of the unknown), since it does not require complex re-meshing when the geometry is modified. Several numerical tests are successfully performed, which asses the effectiveness of the present approach.
\end{abstract}

AMS subject classifications: 74B05, 65N06, 74S20, 74G15

Key words: Linear Elasticity, Cauchy-Navier equations, ground deformation, unbounded domain, coordinate transformation method, Cartesian grid, Ghost points, Level-set methods, Multigrid.

\section{Introduction}

Physics-based models of ground deformation at volcanoes have been very promising for their ability to predict surface displacements from forces acting within the Earth.

*Corresponding author. Email addresses: coco@dmi.unict.it (A. Coco), gilda.currenti@ct.ingv.it (G. Currenti), ciro.delnegro@ct.ingv.it (C. Del Negro), russo@dmi .unict.it (G. Russo) 
By comparing or fitting surface observations to the predictions from these mathematical models, better constraints on important properties of volcanic systems have been inferred [16, 35, 47]. Models based on analytical and semi-analytical solutions of the elasto-static Cauchy-Navier equations are often used to provide a first approximation of the expected surface deformation [27,49]. However, several features, such as irregular geometries (volcano topography and composite source of deformation) and heterogeneous medium properties, cannot be accounted for in analytical formulations. Numerical solutions based on Finite Element and Boundary Element methods have been investigated, showing that these features may significantly affect the solutions (see for example $[8,15,35,43,46,47]$ for deformation computations with realistic geophysical data). Despite the capability to solve deformation models in complex domains, the use of FEM is computationally expensive since the mesh is geometry-dependent and mesh construction requires careful design, testing, and validation to ensure that the configuration leads to an acceptable solution. Therefore, for a complex geometry, generation of a good mesh is not a trivial task and may require a considerable amount of work [31]. On the other hand, Boundary Element methods cannot be employed in problems with heterogeneous media or in presence of source.

In volcanology the elasto-static problem is usually posed in an unbounded (infinite) domain, meaning that the fields extend toward infinity. For solving such a problem, the unbounded domain is typically truncated at a sufficiently large distance from the source and appropriate Artificial Boundaries Conditions (ABCs) have to be imposed on these new external artificial boundaries in such a way that the solution of the truncated model approaches the one of the unbounded medium. This method is used in several fields, such as acoustic, electro-dynamics, solid and fluid mechanics [2, 25,44,48].

Discretizing the truncated domain with a uniform grid usually requires a very large number of grid nodes, making the method rather inefficient. Furthermore, the definition of the appropriate $\mathrm{ABCs}$ is an open problem, since various different approaches have been proposed in the literature $[17,30,33,34]$ and it is not clear what are their relative merits. In some cases, the choice of $\mathrm{ABC}$ is not unique $[22,44,48]$ and strongly affects the solutions. A similar approach has been recently adopted in [39]: the problem is transformed into an infinite system of equations and a careful convergence theory suggests where to perform the truncation.

Another strategy consists of using Quasi-Uniform Meshes (QUM) (see, for instance, [1, 20,29]) that adopts a smooth, strictly monotonic function to map the original unbounded domain into a bounded one, which is then discretized by a uniform mesh. By this approach, the drawbacks of the truncated domain are avoided, since all the infinite domain is taken into account in the mapping. The nodes of QUM are located at mid-point of each cell in order to avoid the numerical issue caused by the last (infinite) spatial step (see Fig. $1)$.

At variance with the classical QUM, we adopt a coordinate transformation method, which consists of using a smooth, strictly monotonic function to transform the original problem (domain, geometry, equations and boundary conditions) into a new problem in 


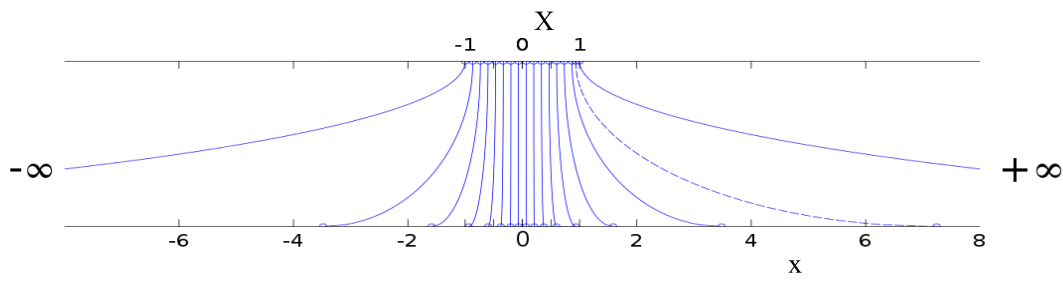

Figure 1: Coordinate mapping from the computational space $X \in[-1,1]$ to real space $x \in[-\infty,+\infty]$. The computational domain is divided into 15 cells. The continuous lines represent the mapping of cell edges. The dashed line represents the mapping of the center of the last cell.

a bounded domain, which is then discretized on a uniform mesh. By this transformation, the boundary conditions at infinity are taken into account in a natural way and a classical finite-difference method on uniform Cartesian grids can be applied to discretize the equations. The coordinate transformation method was firstly proposed in [23] and successively adopted in several contexts: for example, in [3] several methods for solving problems in a semi-infinite or infinite domain using Chebyshev polynomials are analyzed; in [24] some Jacobi approximations for solving differential equation on the half line are investigated; in [32] the transformed finite problem is transcribed to a system of algebraic equations by Chebyshev-Gauss collocation.

All the above techniques have been adopted in one space dimension. There is a vast literature on quasi-uniform grids in several space dimensions, which are based on smooth mapping between the infinite domain and the unit square. As an example we cite the book of J. Castillo [7]. In case of smooth domain these quasi-uniform grid mappings onto the unit square are able to provide discretization of the mapped equations into new coordinates that can be discretized by a regular Cartesian grid, combining the benefit of both efficiency and accuracy, typical of regular Cartesian grids. However this approach may become less effective for complex possibly time dependent geometries, because the mapping becomes very complicated and needs also to be updated in time.

A fast and efficient numerical method, which preserves the advantage of modelling complex structures and overcomes the drawback of meshing procedures, has been recently proposed in [10] in the context of Poisson equation with mixed boundary conditions on bounded domains. The method is based on a second order Multigrid FiniteDifference (FD) ghost-point method for solving the equations in an arbitrary domain described by a level-set function. The problem is discretized on a Cartesian grid and the level set function is used to define the domain geometry. The basic idea of the level-set method, introduced by Osher and Sethian [38], is to represent complex interfaces implicitly as the zero level set of a continuous function [19,37,40-42]. Due to the implicit representation on a Cartesian grid, the level set approach does not introduce any a priori assumptions on the geometry and, therefore, is receiving growing attention in the context of geometric inverse problems [26] and shape optimization [6, and references therein].

We combine the finite-difference level-set method [10] with the smooth mapping of 
the infinite domain into a square for the numerical solution of the Cauchy-Navier equation and apply the procedure for volcanology problems. By this method we discretize the elasto-static operator in the transformed coordinates using central differences, namely using a symmetric nine-point stencil, centered at each internal grid point. Some equations for internal grid points will involve ghost points out of the domain. The equations for such ghost points are obtained by imposing boundary conditions on suitably selected points on the boundary. The linear system arising from the discretization of the problem is solved by a suitable geometric multigrid approach, which is an extension of the multigrid developed in the framework of scalar elliptic equations [10] and a class of systems of PDE's [9].

The plan of the paper is the following. In the next section we recall the mathematical formulation of Cauchy-Navier equations. In the subsequent section, which is the core section of the paper, we describe the numerical method, namely the choice of the coordinate transformation, the finite-difference level-set ghost-point method for the Cauchy-Navier equation in the transformed finite domain, and the geometric multigrid. The last section is devoted to numerical results. The proposed methodology is implemented for the two-dimensional plane-strain elastic model and validated versus exact or analytical solutions, when available. Several numerical tests are performed to show the second order accuracy of the numerical scheme. We also investigate the application of this method to volcanology for computing rock deformation caused by the pressurization of a magma source, with a realistic physical domain obtained by taking a vertical cross section of mount Etna.

\section{Mathematical formulation: Cauchy-Navier Equations}

The deformation and stress fields produced by pressure sources usually occur very slowly, so the medium is in static equilibrium and the displacement can be found by solving the elasto-static equations. In the case where medium behaves elastically, the equations of equilibrium are coupled with constitutive Hooke's law giving the following set of equations [21]:

$$
\left\{\begin{array}{l}
\nabla \cdot \boldsymbol{\sigma}=0 \\
\boldsymbol{\sigma}=\lambda \operatorname{tr}(\boldsymbol{\epsilon}) \boldsymbol{I}+2 \mu \boldsymbol{\epsilon} \\
\boldsymbol{\epsilon}=\frac{1}{2}\left(\nabla \boldsymbol{u}+(\nabla \boldsymbol{u})^{T}\right)
\end{array}\right.
$$

where $\sigma$ and $\boldsymbol{\epsilon}$ are the stress and strain tensors, respectively, $\boldsymbol{u}=(u, v, w)$ the displacement vector and $\lambda$ and $\mu$ are the Lame's elastic medium parameters related to the Young's modulus $E$ and the Poisson ratio $v$ :

$$
\mu=\frac{E}{2(1+v)}, \quad \lambda=\frac{E v}{(1-2 v)(1+v)} .
$$


Without lack of generality, we restrict our attention to the two-dimensional plane strain model, in which we suppose that the $z$ component of displacement $w$ vanishes everywhere, and the displacements $u$ and $v$ are functions of $x$ and $y$ only. The governing equations for the plane-strain model having spatially variable (heterogeneous) material properties are:

$$
\left\{\begin{array}{l}
\frac{\partial}{\partial x}\left((\mu+\lambda) \frac{\partial u}{\partial x}\right)+\nabla \cdot(\mu \nabla u)+\frac{\partial}{\partial x}\left(\lambda \frac{\partial v}{\partial y}\right)+\frac{\partial}{\partial y}\left(\mu \frac{\partial v}{\partial x}\right)=0 \\
\frac{\partial}{\partial y}\left((\mu+\lambda) \frac{\partial v}{\partial y}\right)+\nabla \cdot(\mu \nabla v)+\frac{\partial}{\partial y}\left(\lambda \frac{\partial u}{\partial x}\right)+\frac{\partial}{\partial x}\left(\mu \frac{\partial u}{\partial y}\right)=0
\end{array}\right.
$$
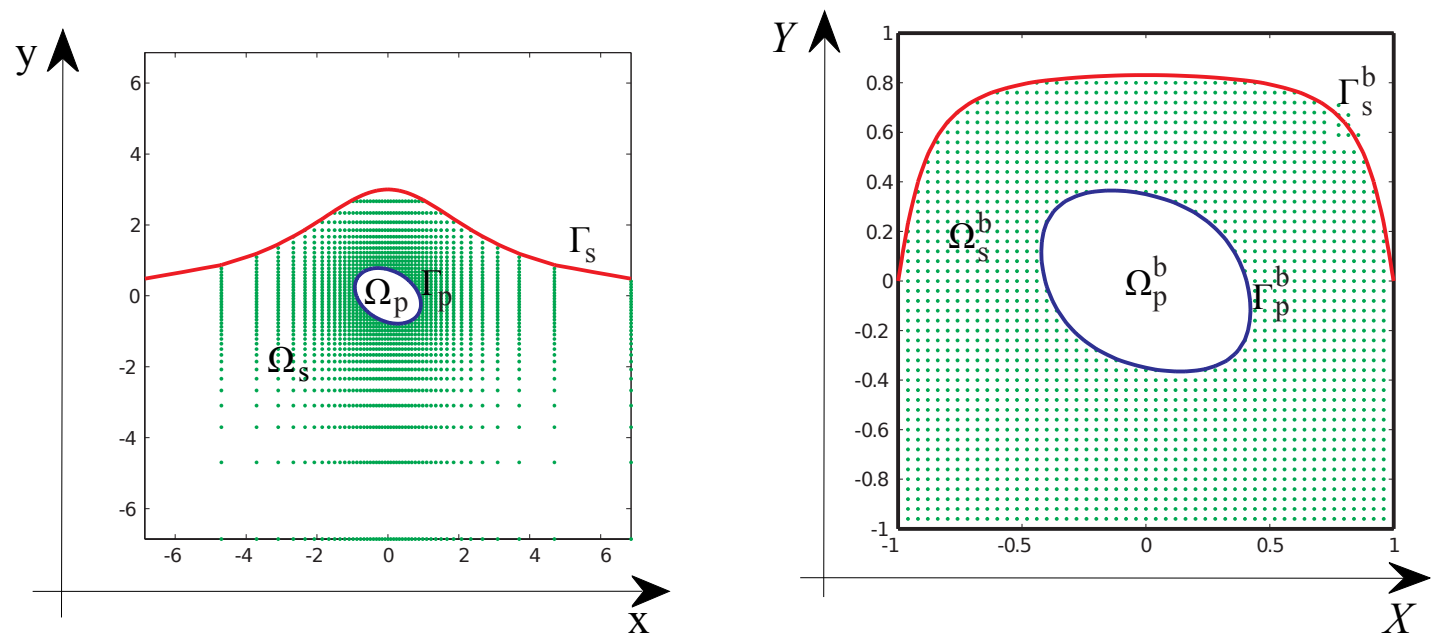

Figure 2: Example of 2D mapping from the bounded (right) to the unbounded (left) domain. The uniform grid for the computational domain (right) automatically results in a quasi-uniform grid for the physical domain (left)

Here, with a slight abuse of notation, we denote by $\nabla$ the two-dimensional gradient operator. The problem is posed in an unbounded domain $\Omega=\Omega_{s} \backslash \Omega_{p}$. The geometry of the problem is represented in Fig. 2 (left), where $\Omega_{p}$ is the region of the pressure source, $\Gamma_{s}$ is the free surface and $\Omega_{s}$ is the domain below the surface $\Gamma_{s}$. The domains $\Omega_{s}$ and $\Omega_{p}$ and the boundaries $\Gamma_{S}$ and $\Gamma_{p}$ are implicitly described by two level-set functions, i.e. [38]:

$$
\Omega_{s}=\left\{\phi_{s}(x, y)<0\right\}, \quad \Omega_{p}=\left\{\phi_{p}(x, y)<0\right\}, \quad \Gamma_{s}=\left\{\phi_{s}(x, y)=0\right\}, \quad \Gamma_{p}=\left\{\phi_{p}(x, y)=0\right\} .
$$

From a level-set function $\phi_{s, p}$ we can easily compute the outward unit normal to the zero level-set and its curvature:

$$
\boldsymbol{n}=\frac{\nabla \phi_{s, p}}{\left|\nabla \phi_{s, p}\right|}, \quad \kappa=\nabla \cdot \boldsymbol{n}
$$


A special case of level-set function is the signed distance function:

$$
\phi_{s}(x, y)=\left\{\begin{array}{rl}
-d\left((x, y), \Gamma_{s}\right) & \text { if }(x, y) \in \Omega_{s} \\
d\left((x, y), \Gamma_{s}\right) & \text { if }(x, y) \notin \Omega_{s}{ }^{\prime}
\end{array} \quad \phi_{p}(x, y)=\left\{\begin{array}{rl}
d\left((x, y), \Gamma_{p}\right) & \text { if }(x, y) \in \Omega_{p}, \\
-d\left((x, y), \Gamma_{p}\right) & \text { if }(x, y) \notin \Omega_{p}
\end{array},\right.\right.
$$

where $d((x, y), \Gamma)$ represents the distance between the point $(x, y)$ and the interface $\Gamma$. A signed distance function is preferred to a simple level-set function because sharp gradients are avoided and it is simpler to compute the boundary closest point to a given grid point. From a general level set function $\phi_{0}$, we can obtain the signed distance function $\phi$ by fast marching methods [41] or by the reinitialization procedure based on the numerical solution of the following PDE (see, for instance, [40])

$$
\frac{\partial \phi}{\partial t}=\operatorname{sgn}\left(\phi_{0}\right)(1-|\nabla \phi|),
$$

for a few time steps.

The problem (2.2) is posed in $\Omega=\Omega_{S} \backslash \Omega_{p}$, with a free-stress boundary condition

$$
\boldsymbol{\sigma} \cdot \boldsymbol{n}_{s}=0 \text { on } \Gamma_{s}
$$

where $n_{s}=\nabla \phi_{s} /\left|\nabla \phi_{s}\right|$ is the unit normal to $\Gamma_{s}$, directed from inside to outside of $\Omega_{s}$, while the condition on the pressure source is

$$
\boldsymbol{\sigma} \cdot \boldsymbol{n}_{p}=-p \boldsymbol{n}_{p} \text { on } \Gamma_{p}
$$

where $\boldsymbol{n}_{p}=\nabla \phi_{p} /\left|\nabla \phi_{p}\right|$ is the unit normal to $\Gamma_{p}$, directed from outside to inside of $\Omega_{p}$, and $p$ is the (given) pressure. The problem is closed imposing zero displacements at infinity.

\section{Numerical method}

We investigate the application of Finite-Difference (FD) ghost-point method for solving the elasto-static equation in unbounded domains.

\subsection{Coordinate transformation method}

We implement a suitable coordinate transformation to map the unbounded domain $\Omega$ to a bounded domain $\Omega^{b}$ and then apply the finite-difference method for the transformed problems in the finite domain. Thereafter we use the uppercase and the lowercase notation for quantities on the bounded and unbounded domain, respectively. In some cases we use the superscript $b$ for referring to quantities in the bounded domain. Let $\chi$ be a differentiable, strictly monotonic function that maps the interval $[-1,1]$ onto $[-\infty,+\infty]$. Then we perform the transformation of the coordinates $x=\chi(X)$ and $y=\chi(Y)$, that 
maps $[-1,1] \times[-1,1]$ onto $[-\infty,+\infty] \times[-\infty,+\infty]$. The quantities in the bounded domain $\mu^{b}, \lambda^{b}, \phi_{s}^{b}, \phi_{p}^{b}$ are defined by

$$
\left(\mu^{b}, \lambda^{b}, \phi_{s}^{b}, \phi_{p}^{b}\right)(X)=\left(\mu, \lambda, \phi_{s}, \phi_{p}\right)(\chi(X)) .
$$

The geometry of the new problem is represented in Fig. 2 (right), where

$\Omega_{s}^{b}=\left\{\phi_{s}^{b}(X, Y)<0\right\}, \quad \Omega_{p}^{b}=\left\{\phi_{p}^{b}(X, Y)<0\right\}, \quad \Gamma_{s}^{b}=\left\{\phi_{s}^{b}(X, Y)=0\right\}, \quad \Gamma_{p}^{b}=\left\{\phi_{p}^{b}(X, Y)=0\right\}$.

The new set of equations is obtained by replacing the differential operators with the following symbolic expressions:

$$
\frac{\partial}{\partial x}=\frac{1}{\chi^{\prime}(X)} \frac{\partial}{\partial X}=: D_{X}, \quad \frac{\partial}{\partial y}=\frac{1}{\chi^{\prime}(Y)} \frac{\partial}{\partial Y}=: D_{Y} .
$$

The new problem then becomes:

$$
\left\{\begin{array}{l}
D_{X}\left(\left(\mu^{b}+\lambda^{b}\right) D_{X} U\right)+\nabla^{b} \cdot\left(\mu^{b} \nabla^{b} U\right)+D_{X}\left(\lambda^{b} D_{Y} V\right)+D_{Y}\left(\mu^{b} D_{X} V\right)=0 \\
D_{Y}\left(\left(\mu^{b}+\lambda^{b}\right) D_{Y} V\right)+\nabla^{b} \cdot\left(\mu^{b} \nabla^{b} V\right)+D_{X}\left(\mu^{b} D_{Y} U\right)+D_{Y}\left(\lambda^{b} D_{X} U\right)=0
\end{array}\right.
$$

in the unknowns $U$ and $V$ of the transformed coordinates $X$ and $Y$, and with $\nabla^{b}:=$ $\left(D_{X}, D_{Y}\right)$. Eqs. (3.1) can be resumed in the compact form

$$
\nabla^{b} \cdot \sigma^{b}=0
$$

where (from (2.1)) $\sigma^{b}=\lambda^{b} \operatorname{tr}\left(\boldsymbol{\epsilon}^{b}\right) \boldsymbol{I}+2 \mu^{b} \boldsymbol{\epsilon}^{\boldsymbol{b}}$, with $\boldsymbol{\epsilon}^{\boldsymbol{b}}=\frac{1}{2}\left(\nabla^{b} \boldsymbol{U}+\left(\nabla^{b} \boldsymbol{U}\right)^{T}\right)$. The free-stress boundary condition (2.3) becomes

$$
\sigma^{b} \cdot \tilde{\boldsymbol{n}}_{s}^{b}=0 \text { on } \Gamma_{s}^{b}
$$

where $\tilde{\boldsymbol{n}}_{s}^{b}=\nabla^{b} \phi_{s}^{b} /\left|\nabla^{b} \phi_{s}^{b}\right|$, while the condition on the pressure source (2.4) reads

$$
\sigma^{b} \cdot \tilde{\boldsymbol{n}}_{\boldsymbol{p}}^{\boldsymbol{b}}=-p^{b} \tilde{\boldsymbol{n}}_{\boldsymbol{p}}^{\boldsymbol{b}} \text { on } \Gamma_{p}^{b}
$$

where $\tilde{\boldsymbol{n}}_{p}^{b}=\nabla^{b} \phi_{p}^{b} /\left|\nabla^{b} \phi_{p}^{b}\right|$. We observe that $\tilde{\boldsymbol{n}}_{s}^{b}$ and $\tilde{\boldsymbol{n}}_{p}^{b}$ are not the normal to $\Gamma_{s}^{b}$ and $\Gamma_{p}^{b}$, respectively, in the computational domain. This because the gradient operator $\nabla^{b}$ is not the classical gradient, due to the non linear mapping.

The Dirichlet boundary conditions imposed at infinity become:

$$
U( \pm 1, Y)=U(X, \pm 1)=V( \pm 1, Y)=V(X, \pm 1)=0 .
$$

The finite domain is evenly discretized by a regular Cartesian grid with $(N+1)^{2}$ grid nodes giving a spatial resolution of $H=2 / N$. The coordinate transformation is especially 
convenient because it straightforwardly offers a non-uniform grid in the infinite domain, with a decreasing spatial resolution going toward infinity (see Fig. 2).

There are several typical mappings that relate infinite and finite domains to each other. Since the analytical (exact) solutions for the elasto-static problem of a pressure source in an infinite half-space domain decays algebraically as $x$ and $y$ go to infinity [27], it is expected that good results are given by an algebraic transformation, like the following:

$$
\chi(X)=\frac{c X}{\left(1-X^{2}\right)^{m}}
$$

where $c$ and $m$ are positive parameters, which define the grid point distribution in the original (physical) infinite domain.

Knowing that the solution of the elasto-static problem (2.2) with boundary conditions $(2.3,2.4)$ decades as $1 / \sqrt{x^{2}+y^{2}}$ going to infinity [27], we choose $m$ in such a way that the asymptotic behavior of the solution is properly captured. This is accomplished by the requirement that the gradient of the transformed solution in the bounded domain does not develop singularity at the boundary of $[-1,1] \times[-1,1]$. Without loss of generality we can perform a one-dimensional computation. The asymptotic behavior of the solution and its derivative are given by:

$$
u(x)=U(X) \sim \frac{1}{x}=\frac{1}{\chi(X)}=\frac{\left(1-X^{2}\right)^{m}}{c X}, \quad \frac{\partial U}{\partial X} \sim \frac{\partial}{\partial X} \frac{\left(1-X^{2}\right)^{m}}{c X}=\frac{m\left(1-X^{2}\right)^{m-1}}{c X}-\frac{\left(1-X^{2}\right)^{m}}{c X^{2}}
$$

which suggest to choose $m \geq 1$ in order to avoid singularity. By increasing $m$, faster spread in the grid node distribution is obtained. Therefore, it is convenient to use $m=1$ in order to not spread out the nodes in a too large domain.

The choice of $c$ regulates the length scale of the computational grid. Since higher gradients in the solution are mainly concentrated around the source $\Omega_{p}$ and decade going to infinity, the finer part (which is also approximately uniform) of the grid should be placed in the vicinity of $\Omega_{p}$. The minimum grid spacing in the unbounded domain is $h_{\min }=H \cdot \chi^{\prime}(0)=2 c / N$ at the origin, near which we place $\Omega_{p}$, without loss of generality. In addition, to ensure a fine resolution in a suitable square $[-\alpha, \alpha]^{2}$ containing the source $\Omega_{p}$, we impose that the spatial resolution at the boundary of $[-\alpha, \alpha]^{2}$ is half the resolution at the origin. We choose $c$ so that this condition is satisfied. In detail, we impose $\chi^{\prime}\left(\chi^{-1}(\alpha)\right)=2 \cdot \chi^{\prime}(0)$. If $m=1$, after some algebra we obtain the condition:

$$
c=\alpha \frac{\sqrt{2}}{2}
$$

Under these assumptions, the mapping function (3.5) reads as:

$$
\chi(X)=\frac{\alpha \sqrt{2}}{2} \frac{X}{\left(1-X^{2}\right)} .
$$




\subsection{Finite-Difference Ghost-Point Method}

We apply the finite-difference ghost-point method of Coco and Russo [10] to the transformed problem (3.1)-(3.4) in the finite domain $[-1,1] \times[-1,1]$. The discretization of the problem leads to a linear system, which is obtained as follows. For each grid point of $\Omega^{b}=\Omega_{s}^{b} \backslash \Omega_{p}^{b}$ we discretize the two equations (3.1) by central differences. Note that all terms of Eqs. (3.1) can be expressed in the following terms:

$$
\frac{\partial}{\partial X}\left(\gamma \frac{\partial W}{\partial X}\right), \frac{\partial}{\partial X}\left(\gamma \frac{\partial W}{\partial Y}\right), \frac{\partial}{\partial Y}\left(\gamma \frac{\partial W}{\partial X}\right), \frac{\partial}{\partial Y}\left(\gamma \frac{\partial W}{\partial Y}\right),
$$

$\gamma$ being a smooth coefficient and $W=U, V$. The discretization of the previous terms reads:

$$
\begin{aligned}
\frac{\partial}{\partial X}\left(\gamma \frac{\partial W}{\partial X}\right) & \approx \frac{\gamma_{i+1 / 2, j}\left(W_{i+1, j}-W_{i, j}\right)-\gamma_{i-1 / 2, j}\left(W_{i, j}-W_{i-1, j}\right)}{H^{2}}, \\
\frac{\partial}{\partial X}\left(\gamma \frac{\partial W}{\partial Y}\right) & =\frac{\partial \gamma}{\partial X} \frac{\partial W}{\partial Y}+\gamma \frac{\partial^{2} W}{\partial X \partial Y} \\
& \approx \frac{\left(\gamma_{i+1, j}-\gamma_{i-1, j}\right)\left(W_{i, j+1}-W_{i, j-1}\right)}{4 H^{2}}+\gamma_{i, j} \frac{W_{i+1, j+1}+W_{i-1, j-1}-W_{i+1, j-1}-W_{i-1, j+1}}{4 H^{2}}, \\
\frac{\partial}{\partial Y}\left(\gamma \frac{\partial W}{\partial X}\right) & =\frac{\partial \gamma}{\partial Y} \frac{\partial W}{\partial X}+\gamma \frac{\partial^{2} W}{\partial X \partial Y} \\
\frac{\partial}{\partial Y}\left(\gamma \frac{\partial W}{\partial Y}\right) & \approx \frac{\left(\gamma_{i, j+1}-\gamma_{i, j-1}\right)\left(W_{i+1, j}-W_{i-1, j}\right)}{4 H^{2}}+\gamma_{i, j} \frac{W_{i+1, j+1}+W_{i-1, j-1}-W_{i+1, j-1}-W_{i-1, j+1}}{4 H^{2}},
\end{aligned}
$$

The whole stencil results in a nine-point stencil. For grid points of $\Omega^{b}$ which are close to the boundary, some of the points of the stencil may lie outside $\Omega^{b}$ (i.e. outside $\Omega_{s}^{b}$ or inside $\Omega_{p}^{b}$ ). Such grid points are called ghost points and a suitable value of $U$ and $V$ should be defined for them to close the linear system, as explained later.

In order to reduce the amount of ghost points, we reduce the nine-point stencil for grid points close to the boundary to a seven-point stencil. In particular, if a grid point $P$ has one of the eight surrounding grid points outside $\Omega^{b}$ (see Fig. 3), then we compute the unit normal $n^{b}(P) \equiv\left(n_{X}^{b}, n_{Y}^{b}\right)=\nabla \phi^{b}(P) /\left|\nabla \phi^{b}(P)\right|$ by central differences, where $\phi^{b}=\phi_{s}^{b}$ or $\phi^{b}=\phi_{p}^{b}$ whether the grid point is closer to $\Gamma_{s}^{b}$ or $\Gamma_{p}^{b}$, respectively. Now, if $\tilde{n}_{X}^{b} \cdot \tilde{n}_{Y}^{b} \geq 0$, we use the following discretization for the mixed derivative ${ }^{\dagger}$ :

$$
\frac{\partial^{2} W}{\partial X \partial Y} \approx \frac{1}{2 H^{2}}\left[\begin{array}{rrr}
-1 & 1 & 0 \\
1 & -2 & 1 \\
0 & 1 & -1
\end{array}\right] W_{i, j}
$$

\footnotetext{
${ }^{+}$with the notation $S w_{i, j}$, where $S=\left(s_{l m}\right), l, m=-1,0,1$, we denote $S w_{i, j}=\sum_{l=-1}^{1} \sum_{m=-1}^{1} s_{l m} w_{i+l, j+m}$
} 
while, if $\tilde{n}_{X}^{b} \cdot \tilde{n}_{Y}^{b}<0$, we use:

$$
\frac{\partial^{2} W}{\partial X \partial Y} \approx \frac{1}{2 H^{2}}\left[\begin{array}{rrr}
0 & -1 & 1 \\
-1 & 2 & -1 \\
1 & -1 & 0
\end{array}\right] W_{i, j} .
$$

See Fig. 3 for a graphic explanation.

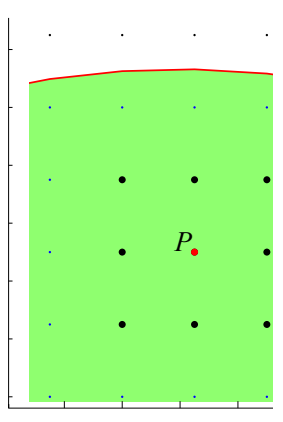

$\frac{1}{4 H^{2}}\left[\begin{array}{rrr}-1 & 0 & 1 \\ 0 & 0 & 0 \\ 1 & 0 & -1\end{array}\right]$

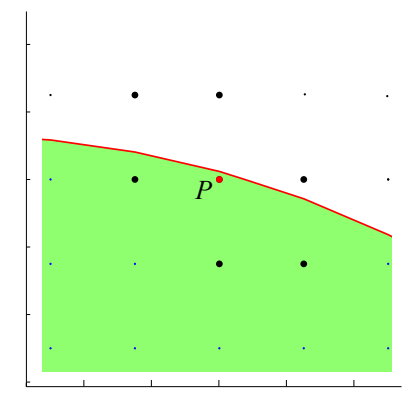

$\frac{1}{2 H^{2}}\left[\begin{array}{rrr}-1 & 1 & 0 \\ 1 & -2 & 1 \\ 0 & 1 & -1\end{array}\right]$

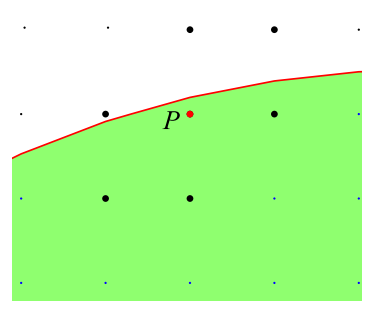

$\frac{1}{2 H^{2}}\left[\begin{array}{rrr}0 & -1 & 1 \\ -1 & 2 & -1 \\ 1 & -1 & 0\end{array}\right]$

Figure 3: The stencil for the mixed derivative changes accordingly to the distance from the boundary and to the normal direction.

We mark all inside and ghost grid points and call them active grid points.

In order to close the linear system, we write an equation for each ghost point. Let $G$ be a ghost point. Therefore $G \notin \Omega_{s}^{b}$ or $G \in \Omega_{p}^{b}$. Let us suppose that $G$ is outside $\Omega_{s}^{b}$ (if $G$ is inside $\Omega_{p}^{b}$ the discretization is analogous). We compute the outward unit normal in $G$, that is $n_{G}^{b}=\nabla \phi_{s}^{b}(G) /\left|\nabla \phi_{s}^{b}(G)\right|$, using a second-order accurate discretization for $\nabla \phi_{s}^{b}(G)$, such as central differences in $G$. Now we can compute the closest boundary point to $G$, that we call $B$, by the level-set function, applying the bisection method to $\phi_{s}^{b}$ along the normal direction $n_{G}^{b}$. Therefore, the two equations of the linear system for the variables $U$ and $V$ in the ghost point $G$ are obtained by the boundary conditions (3.3) and (3.4) on $\Gamma_{s}^{b}$ :

$$
\sigma^{b}(\overline{\boldsymbol{U}}(B)) \cdot \bar{n}^{b}(B)=0, \text { with } \bar{n}^{b}(B)=\left(\bar{n}_{X}^{b}, \bar{n}_{Y}^{b}\right)=\frac{\nabla^{b} \bar{\phi}_{s}^{b}(B)}{\left|\nabla^{b} \bar{\phi}_{s}^{b}(B)\right|^{\prime}},
$$

where $\bar{U}$ and $\bar{\phi}_{s}^{b}$ are the biquadratic interpolants of $\boldsymbol{U}$ and $\phi_{s}^{b}$ respectively on a suitable upwind nine-point stencil (which is described later) made by active grid points. Let us recall that a biquadratic interpolant of a function $f(x, y)$ is a polynomial $\bar{f}(x, y)=\sum_{i, j=0}^{2} a_{i, j} x^{i} y^{j}$ where the nine coefficients $a_{i, j}$ are determined imposing $f=\bar{f}$ in the nine points of the stencil. 
The boundary conditions (3.7) lead to two scalar equations:

$$
\begin{aligned}
& \left(\frac{2 \mu^{b}+\lambda^{b}}{\chi^{\prime}(X)} \frac{\partial \bar{U}}{\partial X}+\frac{\lambda^{b}}{\chi^{\prime}(Y)} \frac{\partial \bar{V}}{\partial Y}\right) \bar{n}_{X}^{b}+\mu^{b}\left(\frac{1}{\chi^{\prime}(Y)} \frac{\partial \bar{U}}{\partial Y}+\frac{1}{\chi^{\prime}(X)} \frac{\partial \bar{V}}{\partial X}\right) \bar{n}_{Y}^{b}=0 \\
& \mu^{b}\left(\frac{1}{\chi^{\prime}(Y)} \frac{\partial \bar{U}}{\partial Y}+\frac{1}{\chi^{\prime}(X)} \frac{\partial \bar{V}}{\partial X}\right) \bar{n}_{X}^{b}+\left(\frac{2 \mu^{b}+\lambda^{b}}{\chi^{\prime}(Y)} \frac{\partial \bar{V}}{\partial Y}+\frac{\lambda^{b}}{\chi^{\prime}(X)} \frac{\partial \bar{U}}{\partial X}\right) \bar{n}_{Y}^{b}=0 .
\end{aligned}
$$

We use the first condition to write the linear equation of the linear system for the unknown $U(G)$ and the second equation for $V(G)$.
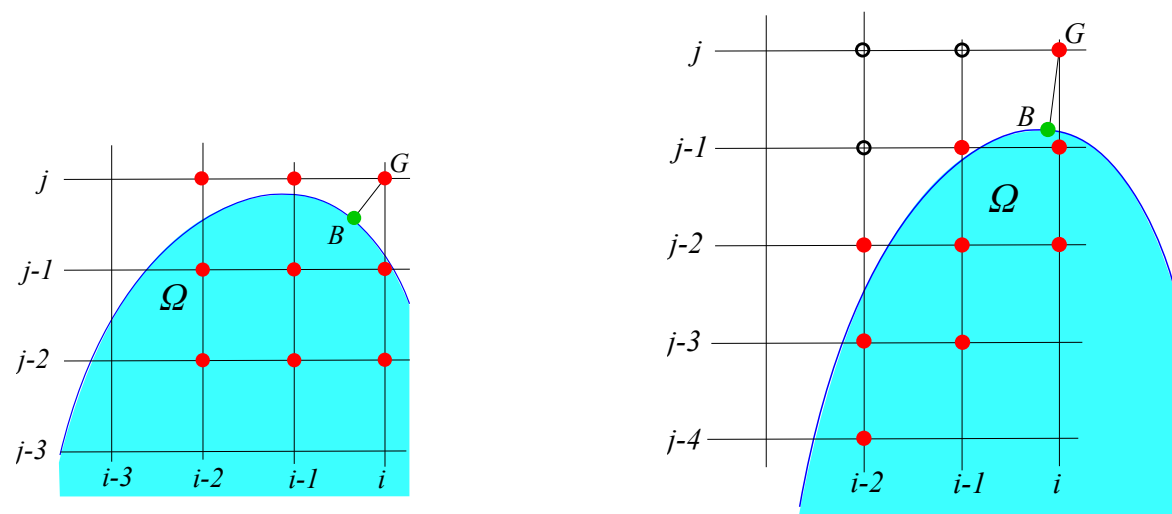

Figure 4: Upwind nine-point stencil associated to a ghost point G. Left: the nine points are the vertices of the $3 \times 3$ square grid whose upper-right corner is $G$. Right: the empty circular grid points are not active points, and therefore are not used in the definition of the stencil, which is instead defined by the nine full circular nodes.

The upwind nine-point stencil is chosen, as described in [10], in the following manner (see Fig. 4 for the case in which both components of the vector $G-B$ are positive, the other three cases are analogous). If $\left|x_{B}-x_{G}\right|<\left|y_{B}-y_{G}\right|$ (as in Fig. 4), the nine-point stencil will be composed by three points of the column $i$, three points of the column $i-s$, three points of the column $i-2 s$, where $s=\operatorname{sgn}\left(x_{B}-x_{G}\right)$; while if $\left|x_{B}-x_{G}\right| \geq\left|y_{B}-y_{G}\right|$ it will be composed by three points of the row $j$, three points of the row $j-s$, three points of the row $j-2 s$, where $s=\operatorname{sgn}\left(y_{B}-y_{G}\right)$. Let us suppose $\left|x_{B}-x_{G}\right|<\left|y_{B}-y_{G}\right|$ and $x_{B}-x_{G}<0$, as in Fig. 4 (the other cases are treated similarly). Then:

- The three points of the column $i$ are those with indices:

$$
(i, j),(i, j-1),(i, j-2) \text {. }
$$

Since the grid point with indices $(i, j-1)$ is an interior point, such three points are active grid points.

- The three points of the column $i-1$ are those with indices:

$$
(i-1, j),(i-1, j-1),(i-1, j-2)
$$


if all of them are active grid points, otherwise we choose those with indices:

$$
(i-1, j-1),(i-1, j-2),(i-1, j-3)
$$

if all of them are active grid points, otherwise we reduce the stencil as described later.

- The three points of the column $i-2$ are those with indices:

$$
(i-2, j),(i-2, j-1),(i-2, j-2)
$$

if all of them are active grid points, otherwise we choose those with indices:

$$
(i-2, j-1),(i-2, j-2),(i-2, j-3)
$$

if all of them are active grid points, otherwise, if the three points for the column $i-1$ were those indicated in (3.9), we choose those with indices:

$$
(i-2, j-2),(i-2, j-3),(i-2, j-4)
$$

if all of them are active grid points, otherwise we reduce the stencil as described later.

We observe that, if the grid is fine enough with respect to the curvature of the boundary, the most common stencil used is the $3 \times 3$ square grid whose upper-right corner is $G$ (left plot of Fig. 4).

If it is not possible to build the nine-point stencil, we revert to a more robust (less accurate) three-point stencil. For example, in the case illustrated in Fig. 5 we choose the grid points with indices:

$$
(i, j),(i, j-1),(i-1, j-1)
$$

and we use a linear interpolation instead of a biquadratic one. These three points are active grid points, since the grid point with indices $(i, j-1)$ is an interior point. Notice that, if the boundary is sufficiently smooth (say $C^{1}$ ), the reduction of the stencil can be avoided provided the grid is sufficiently fine with respect to the curvature. For large variations in the curvature this may result in an expensive numerical method, since only a small part needs to be treated by a small spatial step. This drawback can be overcome by the usage of adaptive grids, adopting a refinement criterion reletad to the curvature of the boundary. The Adaptive Mesh Refinement method (see, for instance, [36]), however, is not the focus of the present paper. Anyway, we experienced in all numerical tests presented in the paper that the stencil reduction occurs only in $O(1)$ ghost points and the whole order of accuracy is not degraded [10]. If the boundary is only Lipschitz continuous, adaptivity on sharp points could not avoid the stencil reduction. Anyway, the solution may present singular gradients (due to the lack of regularity of the boundary, even if boundary conditions and sources are smooth). In this case the proper accuracy order of the method 
cannot be reached. Finally, if the solution is smooth in spite of the boundary singularities, the discretization reduces to first order accuracy where the stencil reductions occur. In this case, in order to maintain a uniform error on the whole domain, one can adopt an adaptivity strategy using a smaller spatial step $H_{s} \ll H$ on the singularities such that $H_{s} \approx H^{2}$. A similar strategy in a different context has been adopted in [11].

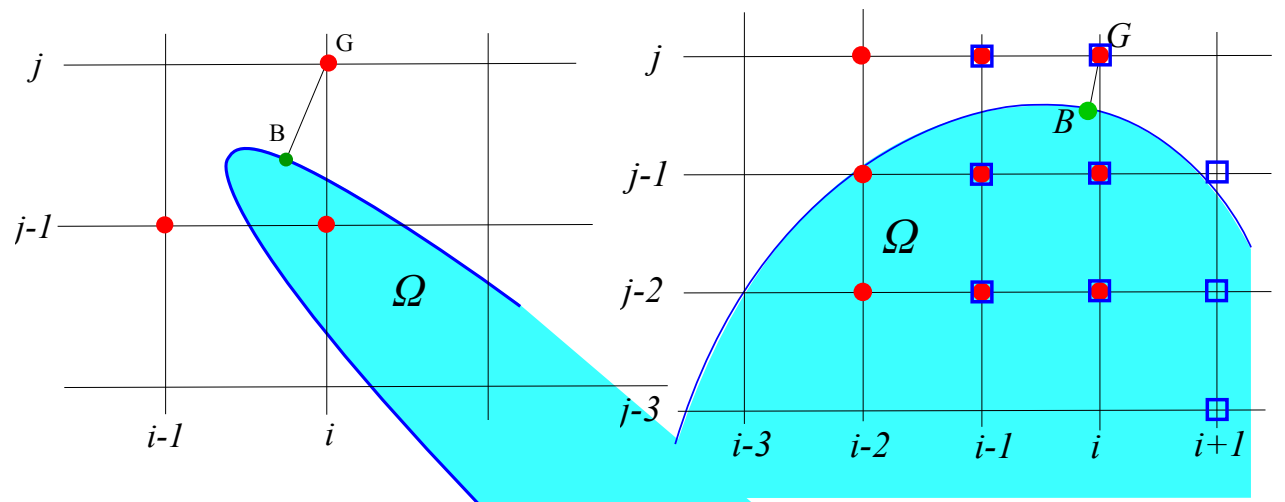

Figure 5: Reduction of the nine point stencil to a three-point stencil.

Figure 6: The upwind stencil is composed by red circular grid points and is used to compute normal derivatives; the central-upwind stencil is composed by blue empty square grid points and is used to compute tangential derivatives.

Let us now introduce the central-upwind nine-point stencil, which, as we will see in the numerical tests of Secs. 4.1 and 4.2, improves the robustness of the method and avoids oscillations in the solution due to a bad conditioning of the coefficient matrix, especially in regions where the geometry is almost flat. The central-upwind stencil is chosen as follows (see Fig. 6, where both the upwind and central-upwind nine-point stencil are plotted). If $\left|x_{B}-x_{G}\right|<\left|y_{B}-y_{G}\right|$ it is composed by three points of the column $i-1$, three points of the column $i$, three points of the column $i+1$; while if $\left|x_{B}-x_{G}\right| \geq\left|y_{B}-y_{G}\right|$ it will be composed by three points of the row $j-1$, three points of the row $j$, three points of the row $j+1$. Let us suppose $\left|x_{B}-x_{G}\right|<\left|y_{B}-y_{G}\right|$ and $x_{B}-x_{G}<0$, as in Fig. 6 (the other cases are treated similarly). Then:

- The three points of the column $i$ are those with indices:

$$
(i, j),(i, j-1),(i, j-2) \text {. }
$$

Since the grid point with indices $(i, j-1)$ is an interior point, such three points are active grid points.

- The three points of the column $i-1$ are those with indices:

$$
(i-1, j),(i-1, j-1),(i-1, j-2)
$$


if all of them are active grid points, otherwise we choose those with indices:

$$
(i-1, j-1),(i-1, j-2),(i-1, j-3)
$$

if all of them are active grid points, otherwise we reduce the stencil as described later.

- The three points of the column $i+1$ are those with indices:

$$
(i+1, j),(i+1, j-1),(i+1, j-2)
$$

if all of them are active grid points, otherwise we choose those with indices:

$$
(i+1, j-1),(i+1, j-2),(i+1, j-3)
$$

if all of them are active grid points, otherwise we reduce the stencil as described later.

If it is not possible to build the nine-point stencil, we revert to a more robust (less accurate) three-point stencil, which is the same described above for the upwind nine-point stencil.

Now, we use the upwind stencil to compute normal derivatives, and the centralupwind stencil to compute tangential derivatives. Therefore, in the first boundary condition of (3.8) we choose the upwind stencil for $U$ and the central-upwind stencil for $V$, while in the second boundary condition of (3.8) we choose the upwind stencil for $V$ and the central-upwind stencil for $U$.

The final system takes the form $A \cdot \boldsymbol{U}=b$, where $\boldsymbol{U}=\left(U_{I}, V_{I}, U_{G}, V_{G}\right), I$ denotes the set of inner points and $G$ the set of ghost points. Matrix $A$ represents the discretization of the equations and the boundary conditions. The linear system is solved by an efficient iterative method based on a geometric multigrid approach, described in the following section.

\subsection{Geometric Multigrid Method}

To solve the sparse linear system $A \cdot \boldsymbol{U}=b$ arising from the discretization of the problem, we adopt an efficient geometric multigrid method by extending a recent approach developed for scalar elliptic problems in [10] and for a class of systems of PDE's in [9].

We briefly resume the main components of this multigrid approach, namely the relaxation scheme and the transfer operators (for more details on classical multigrid see, for instance, [45] or [5]).

The relaxation scheme is obtained by a suitable discretization in space and time of the following fictitious time dependent problem (from (3.2), (3.3) and (3.4)): 


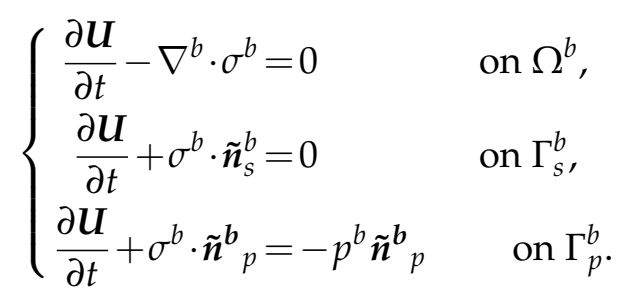

Discretizing the first Eq. of (3.10) in inside grid points $(i, j)$ by forward Euler in time and central differences in space we obtain the following iterative scheme:

$$
\begin{aligned}
& U_{i j}^{(n+1)}=U_{i j}^{(n)}+\Delta t_{1}\left[\nabla_{h}^{b} \cdot \sigma_{h}^{b}\left(U^{(n)}, V^{(n)}\right)\right]_{i j}^{1} \\
& V_{i j}^{(n+1)}=V_{i j}^{(n)}+\Delta t_{2}\left[\nabla_{h}^{b} \cdot \sigma_{h}^{b}\left(U^{(n)}, V^{(n)}\right)\right]_{i j}^{2}
\end{aligned}
$$

where $\left[\nabla_{h}^{b} \cdot \sigma_{h}^{b}\left(U^{(n)}, V^{(n)}\right)\right]_{i j}^{1}$ and $\left[\nabla_{h}^{b} \cdot \sigma_{h}^{b}\left(U^{(n)}, V^{(n)}\right)\right]_{i j}^{2}$ are the two components of the vector $\left[\nabla_{h}^{b} \cdot \sigma_{h}^{b}\left(U^{(n)}, V^{(n)}\right)\right]_{i j}$, which represents the discretization of (3.1) (Sec. 3.2) in the grid node with indices $(i, j)$. Time steps $\Delta t_{1}$ and $\Delta t_{2}$ (which depend on $i$ and $j$ ) are chosen in such a way the iterations (3.11) revert to the Jacobi iteration scheme applied to the respective equations of the linear system $A \cdot U=b$. This is accomplished by choosing $\Delta t_{1}=1 / c_{i j}^{U}$ and $\Delta t_{2}=1 / c_{i j}^{V}$, where $c_{i j}^{U}$ (resp. $c_{i j}^{V}$ ) is the coefficient of the discretization of $\left[\nabla^{b} \cdot \sigma^{b}(U, V)\right]_{1}$ (resp. $\left.\left[\nabla^{b} \cdot \sigma^{b}(U, V)\right]_{2}\right)$ with respect to the unknown $U_{i j}$ (resp. $\left.V_{i j}\right)$.

Discretizing the second Eq. of (3.10) in ghost points $(i, j)$ related to the boundary $\Gamma_{s}^{b}$ by forward Euler in time and upwind or central-upwind scheme in space we obtain the following iterative scheme:

$$
\begin{aligned}
& U_{i j}^{(n+1)}=U_{i j}^{(n)}+\Delta t\left[\sigma_{h}^{b}\left(U^{(n)}, V^{(n)}\right) \cdot \tilde{\boldsymbol{n}}_{s}^{b}\right]_{i j}^{1} \\
& V_{i j}^{(n+1)}=V_{i j}^{(n)}+\Delta t\left[\sigma_{h}^{b}\left(U^{(n)}, V^{(n)}\right) \cdot \tilde{\boldsymbol{n}}_{s}^{b}\right]_{i j}^{2}
\end{aligned}
$$

where $\left[\sigma_{h}^{b}\left(U^{(n)}, V^{(n)}\right) \cdot \tilde{\boldsymbol{n}}_{s}^{b}\right]_{i j}^{1}$ and $\left[\sigma_{h}^{b}\left(U^{(n)}, V^{(n)}\right) \cdot \tilde{\boldsymbol{n}}_{s}^{b}\right]_{i j}^{2}$ are the two components of the vector $\left[\sigma_{h}^{b}\left(U^{(n)}, V^{(n)}\right) \cdot \tilde{\boldsymbol{n}}_{s}^{b}\right]_{i j}$, which represents the discretization of (3.3) (Sec. 3.2) in the grid node with indices $(i, j)$. Following the idea of [10], we choose a spatial step $\Delta t$ in such a way it satisfies a suitable CFL condition. In the numerical test we experienced that a spatial step satisfying $\Delta t<H /\left(4 \mu_{i j} \cdot \max \left\{\chi^{\prime}\left(X_{i j}\right), \chi^{\prime}\left(Y_{i j}\right)\right\}\right)$ is sufficient to ensure the convergence of the iterative scheme (where $\mu_{i j}, X_{i j}$ and $Y_{i j}$ are the quantities $\mu, X$ and $Y$ computed at the grid point with indices $(i, j))$.

The same argument applies to the third Eq. of (3.10). 
Jacobi scheme is known to have poor smoothing properties and thus is not suitable as a relaxation scheme for the multigrid approach [45]. For this reason we switch from the Jacobi to the Gauss-Seidel iteration scheme. In formulas, using the lexicographic order, we apply the iterative scheme

$$
\begin{aligned}
& U_{i j}^{(n+1)}=U_{i j}^{(n)}+\Delta t_{1}\left[\nabla_{h}^{b} \cdot \sigma_{h}^{b}(\tilde{U}, \tilde{V})\right]_{i j}^{1} \\
& V_{i j}^{(n+1)}=V_{i j}^{(n)}+\Delta t_{2}\left[\nabla_{h}^{b} \cdot \sigma_{h}^{b}(\tilde{U}, \tilde{V})\right]_{i j}^{2}
\end{aligned}
$$

instead of (3.11), and the iterative scheme

$$
\begin{aligned}
& U_{i j}^{(n+1)}=U_{i j}^{(n)}+\Delta t\left[\sigma_{h}^{b}(\tilde{U}, \tilde{V}) \cdot \tilde{\boldsymbol{n}}_{s}^{b}\right]_{i j}^{1}, \\
& V_{i j}^{(n+1)}=V_{i j}^{(n)}+\Delta t\left[\sigma_{h}^{b}(\tilde{U}, \tilde{V}) \cdot \tilde{\boldsymbol{n}}_{s}^{b}\right]_{i j}^{2},
\end{aligned}
$$

instead of (3.12). Here, $\tilde{U}_{l m}=U_{l m}^{(n)}$ and $\tilde{V}_{l m}=V_{l m}^{(n)}$ if the grid node $(i, j)$ precedes $(l, m)$ in the chosen ordering, while $\tilde{U}_{l m}=U_{l m}^{(n+1)}$ and $\tilde{V}_{l m}=V_{l m}^{(n+1)}$ otherwise.

As pointed out in [4] and [10], the efficiency of the multigrid depends mainly on the iterations on inner grid points, since the affection of the convergence factor by the boundary condition iterations can be eliminated with a proper treatment, such as adding some extra-relaxations on and close to the boundary (see [10]).

The transfer (restriction and interpolation) operators are chosen in order to take into account the complex structure of the ghost points, maintaining the simplicity given by the Cartesian grid.

Briefly, the restriction operator is chosen in such a way it does not mix the contribution of inner and ghost residuals, while the interpolation operator is the standard bilinear interpolation. A detailed description of the these transfer operators can be found in [10].

The multigrid is then performed by iterating a $W$-cycle algorithm, choosing the coarsest grid heuristically in such a way that the geometical properties of the domain are still correctly captured (in practice we experienced convergence of the method with a coarsest grid of $17^{2}$ grid points). The $W$-cycle is iterated until a stopping criterion on the residual is satisfied.

\section{Numerical results}

In this section we present several tests aimed to asses the effectiveness of the method. The first test is performed on the whole plane $\mathbb{R}^{2}$ and the results are compared to the exact analytical solution. The second test is in a half-space domain and illustrates the spurious oscillations arising if only the upwind stencil is used and the influence of the $m$ parameter of the mapping function (3.5) in the numerical solution. The last test is performed with a realistic profile of a vertical cross section of mount Etna. 
For each test we compute the relative error in the $L^{\infty}$ and $L^{2}$ norm as follows:

$$
\|E\|_{2}=\left(\frac{\sum_{\left(x_{i}, y_{j}\right) \in \Omega} \mid\left(q_{i, j}-\left.q^{r e f}\left(x_{i}, y_{j}\right)\right|^{2}\right.}{\sum_{\left(x_{i}, y_{j}\right) \in \Omega}\left|q^{r e f}\left(x_{i}, y_{j}\right)\right|^{2}}\right), \quad\|E\|_{\infty}=\frac{\max _{\left(x_{i}, y_{j}\right) \in \Omega}\left|q_{i, j}-q^{r e f}\left(x_{i}, y_{j}\right)\right|}{\max _{\left(x_{i}, y_{j}\right) \in \Omega}\left|q^{r e f}\left(x_{i}, y_{j}\right)\right|},
$$

where $q=u, v$ and $q^{\text {ref }}$ is a reference solution that is the analytical solution when available or a numerical solution computed with another numerical method. The tests are performed in a Cartesian grid composed of $(N+1) \times(N+1)$, whose grid nodes are distributed on the basis of the algebraic mapping function (3.6) using $m=1$ and $\alpha=20 \mathrm{~km}$ to ensure a fine grid resolution near to the source $\Gamma_{p}$. For each test different simulations have been performed varying the number of grid points, i.e. the grid parameter $N$, in order to show that the method is second order accurate.

\subsection{Infinite domain $\Omega=\mathbb{R}^{2} \backslash \Omega_{p}$}
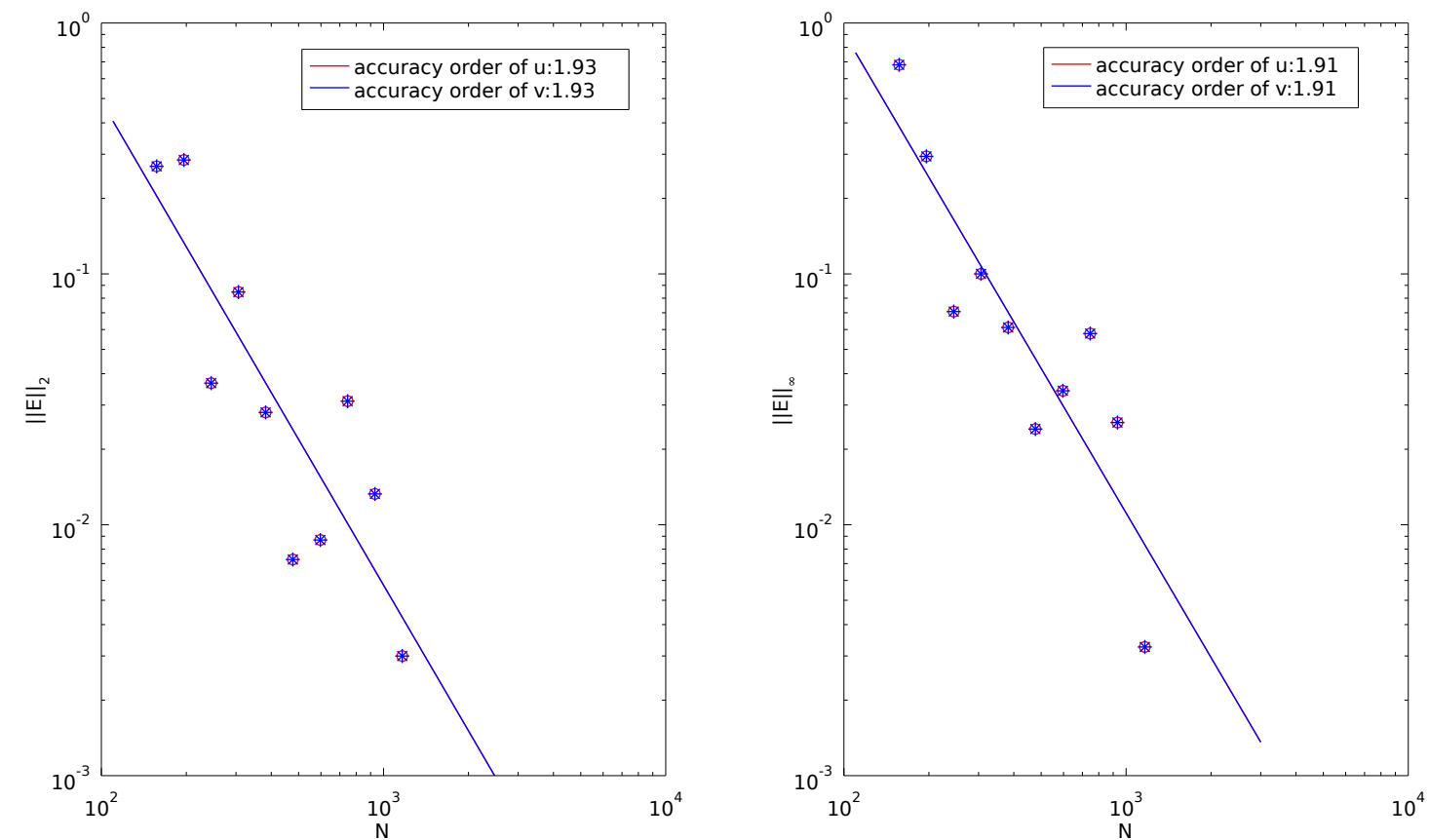

Figure 7: Example 4.1. Bestfit of the $L^{2}$ (left) and $L^{\infty}$ (right) errors of $u$ (star) and $v$ (circle) using only the upwind stencil. The errors for $u$ and $v$ are equals because of the symmetries of the problem and the discretization.

We first consider an infinite domain without surface $\Gamma_{s}$, i.e. with $\phi_{s}=-1$, and a circular pressure source centered at the origin, i.e. $\phi_{p}=\sqrt{x^{2}+y^{2}}-R$, with $R=0.5 \mathrm{~km}$. Analytical solutions of the deformation field have already been devised for this simple geome- 

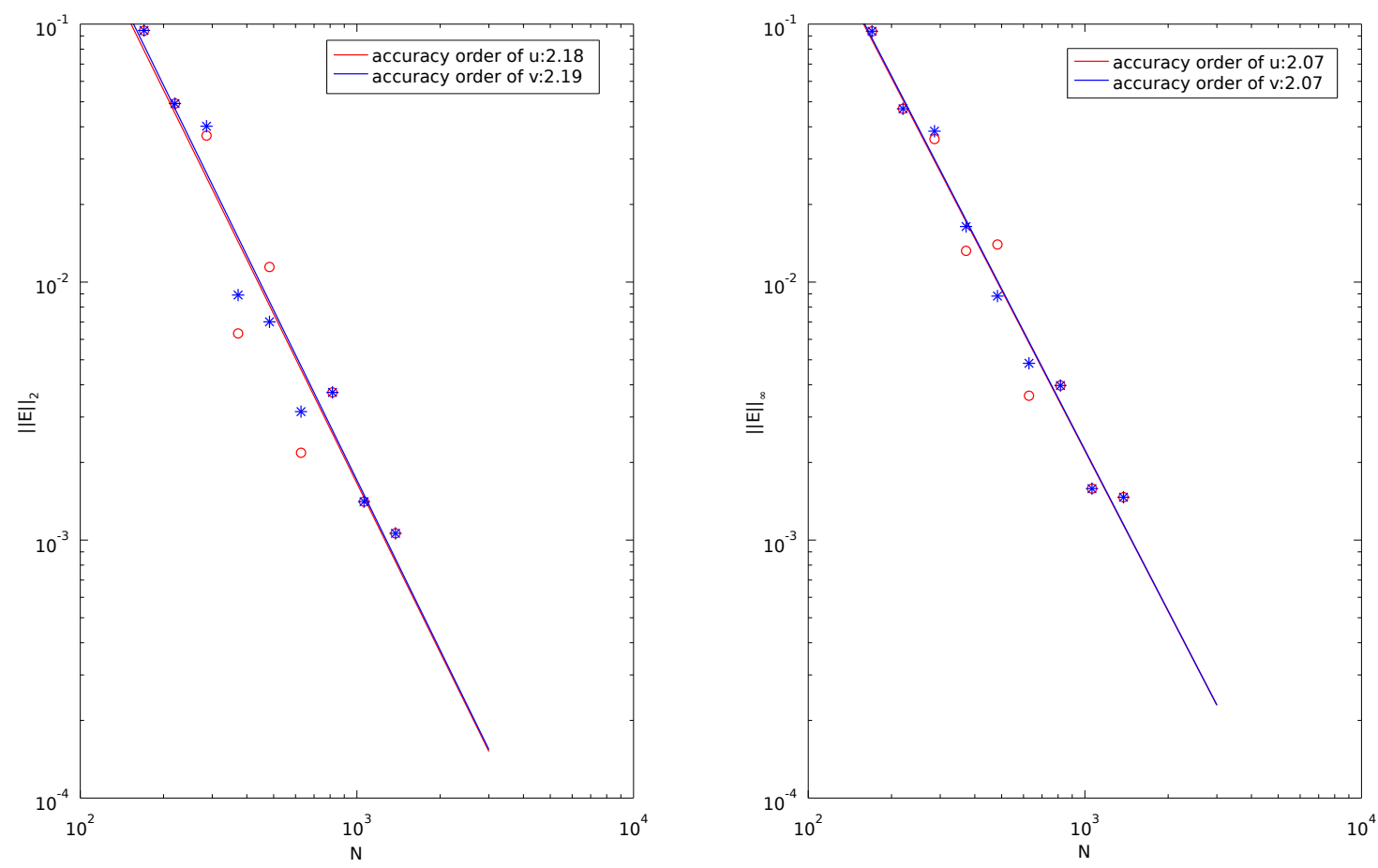

Figure 8: Example 4.1. Bestfit of the $L^{2}$ (left) and $L^{\infty}$ (right) errors of $u$ (star) and $v$ (circle) using the central upwind stencil.

try [27] and can be used to validate the method. The equations of the analytical solutions for the displacements $u$ and $v$ in the unbounded domain are given by:

$$
u=\frac{p R^{2}}{2 \mu} \frac{x}{x^{2}+y^{2}}, \quad v=\frac{p R^{2}}{2 \mu} \frac{y}{x^{2}+y^{2}},
$$

where the source exerts a pressure of $p=10 \mathrm{MPa}$. Elastic properties are set to homogeneous values over the whole domain using a rigidity modulus of $\mu=30 \mathrm{GPa}$. The error is computed as the difference between the analytical and the finite-difference solutions. We first perform the simulation using only the central upwind stencil described in Sec. 3.2. The relative errors in $L^{2}$ and $L^{\infty}$ norms are plotted against $N$ in logarithmic scale in Fig. 7, which shows the second order accuracy. The errors in $u$ and $v$ are equal, due to the symmetry of the problem (Eq. (4.1)) and the discretization. However, the method does not seem sufficiently robust and the errors fluctuate around the best fit line. A possible explanation is that two subsequent grids have almost the same number of grid points. Then, the accuracy of the local approximation oscillates due to interpolation errors in points that are not grid nodes. Such error decreases on average when the grid is refined, but may fluctuate for grids with almost the same number of points leading to a nonmonotone behavior. Another cause is due to the use of the upwind stencil to compute tangential derivatives. In fact, the convergence rate is improved and the fluctuations 
have considerably decreased when the central-upwind stencil is adopted for the tangential derivatives (see Fig. 8). Notice that in this case the errors in $u$ and $v$ are not equal, because of the asymmetry introduced by the central-upwind discretization. Moreover, the main source of the error in the computation of the numerical solution is due to interpolation error near the boundary, when defining the equations for the ghost points. To verify that this is indeed the case, we implemented a numerical scheme which is second order in the bulk, but adopts third order interpolation at the boundary. In the simplest case, this is obtained by bicubic interpolation. The results on the same test problem are reported in Fig. 9. Comparing with Fig.8, we observe that the error is quite smaller and the fluctuations are drasticallv reduced.
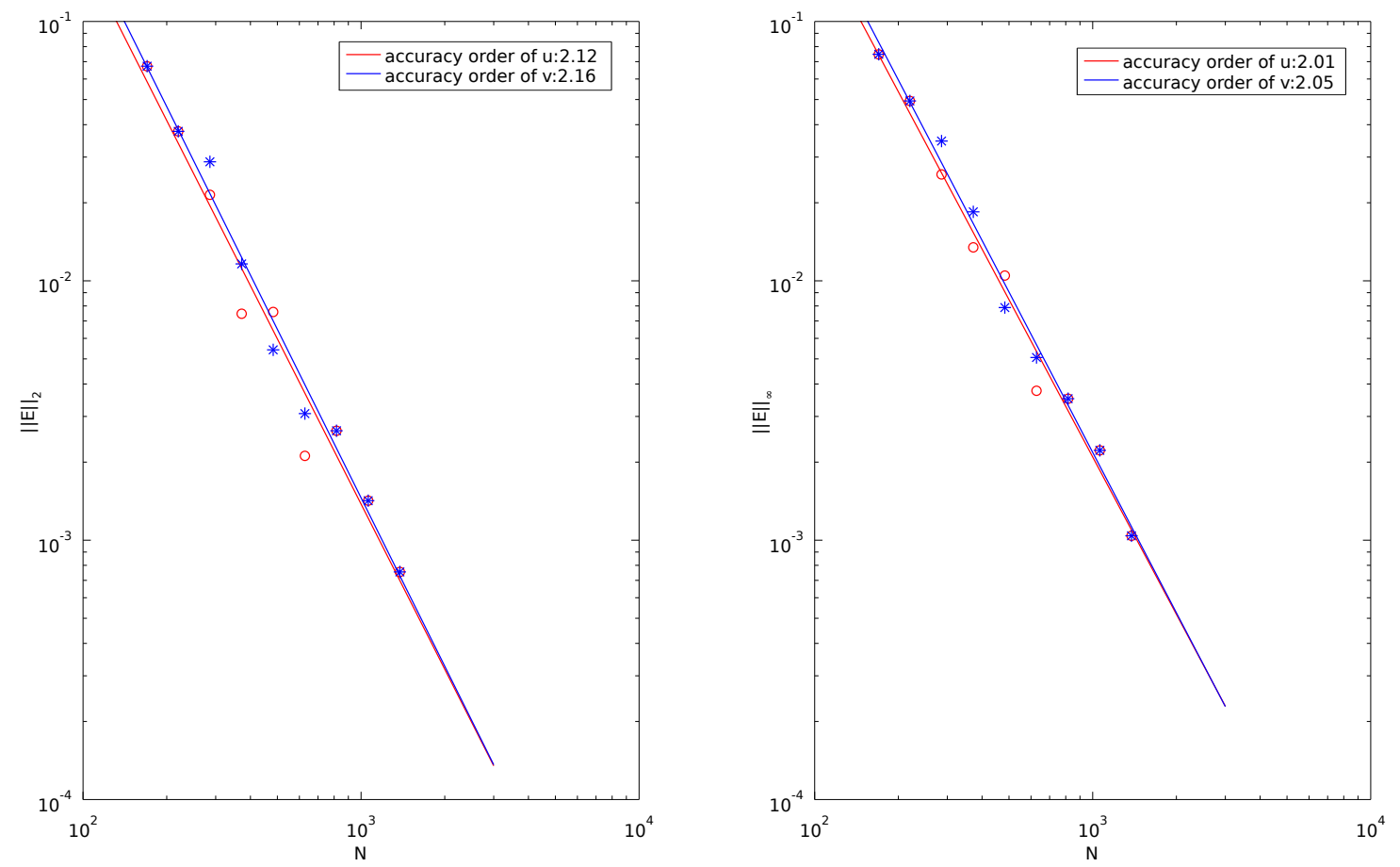

Figure 9: Example 4.1. Bestfit of the $L^{2}$ (left) and $L^{\infty}$ (right) errors of $u$ (star) and $v$ (circle) using the central upwind stencil and a third order accurate discretization of the boundary conditions.

\subsection{Half-space domain}

A further test was set up for computing the deformation caused by a pressure source embedded in a half-space. The boundary $\Gamma_{s}$ is represented by a horizontal line, i.e. $\phi_{s}=$ $y$, while the pressure source is a circle, i.e. $\phi_{p}=\sqrt{x^{2}+\left(y-y_{0}\right)^{2}}-R$, with $y_{0}=-3 \mathrm{~km}$ and $R=0.5 \mathrm{~km}$. Using the upwind scheme, oscillations in the solutions occur along the $\Gamma_{s}$ boundary. This issue is effectively overcome by using the central-upwind scheme 

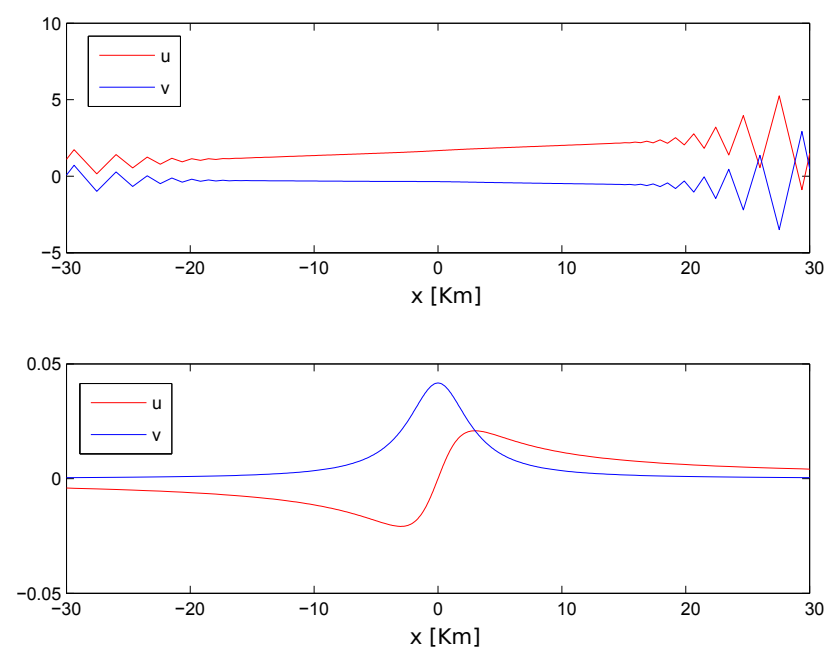

Figure 10: Example 4.2. The usage of the upwind stencil for both $u$ and $v$ gives rise to oscillations on the horizontal surface (top), while the introduction of the central-upwind stencil produces a stable solution (bottom).
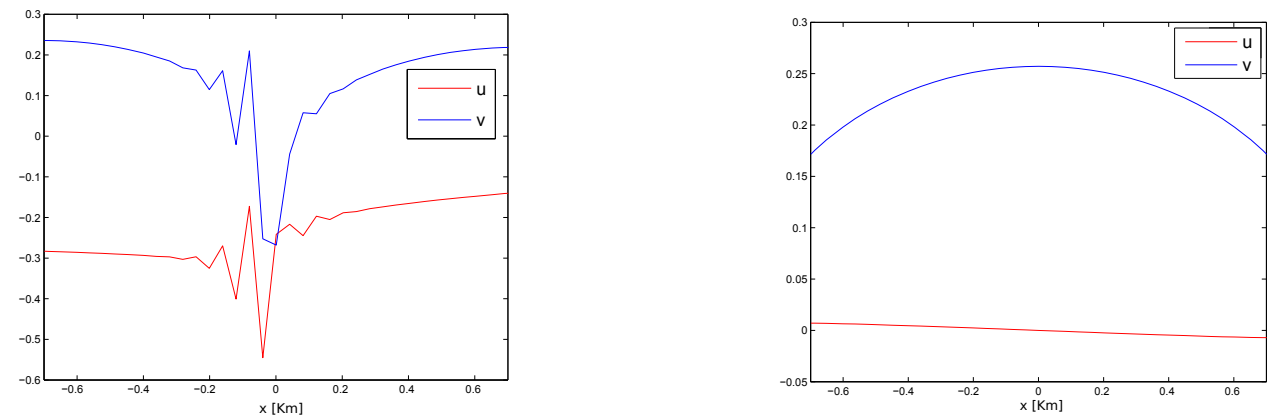

Figure 11: Example 4.2. The usage of the upwind stencil for both $u$ and $v$ gives rise to oscillations on the horizontal profile of the elliptic source of deformation (left), while the introduction of the central-upwind stencil produces a stable solution (right).

proposed in Sec. 3.2 (Fig. 10).

Indeed, the upwind scheme fails to produce acceptable results in regions characterized by a flat surface. Similar oscillations may also occur along $\Gamma_{p}$ whenever part of the boundary is almost flat. For example, this occurs when the boundary $\Gamma_{p}$ is replaced by an ellipse with a higher aspect ratio between the major and the minor axis. We choose $\phi_{p}=x^{2} / a^{2}+\left(y-y_{0}\right)^{2} / b^{2}-1$, with $y_{0}=-3 \mathrm{~km}, a=0.9 \mathrm{~km}$ and $b=0.3 \mathrm{~km}$. As expected, oscillations in the solutions are produced along the flat part of the $\Gamma_{p}$ boundary if the upwind scheme is used. The solutions are stabilized simply using the central-upwind scheme (Fig. 11). No exact analytical solutions are available to verify the accuracy of these results. However, an approximate solution for a spherical source has been devised 

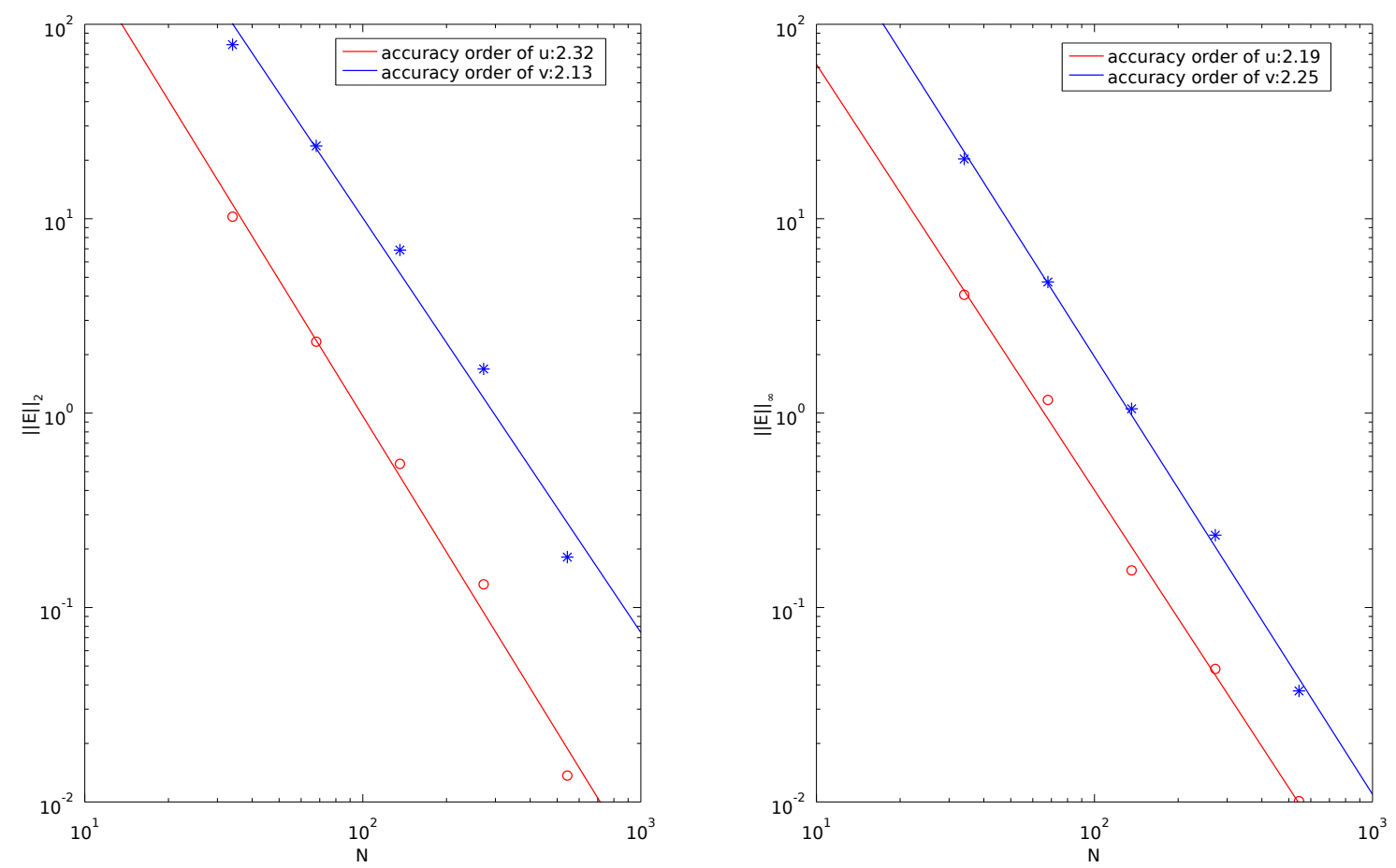

Figure 12: Example 4.2. Bestfit of the $L^{2}$ (left) and $L^{\infty}$ (right) errors of $u$ and $v$ for the spherical pressure source in a half-space domain.

in [49], which estimates the deformation with an error of the order $\left(R / y_{0}\right)^{2}$. The $L^{2}$ and $L^{\infty}$ norms of the relative errors (computed as the difference between the analytical and numerical solutions) for different values of $N$ are plotted in Fig. 12, showing the second order accuracy.

Now, we perform the test for the spherical pressure source with different values of the $m$ parameter of the mapping function (3.5) in order to validate the argument of Sec. 3.1 about its choice. In Fig. 13 we plot the numerical solution of the horizontal displacement $U$ on the bounded domain $\Omega^{b}$ along the surface $\Gamma_{s}^{b}$ computed with $m=0.5, m=1$ and $m=2$. We confirm the argument of Sec. 3.1, i.e. with $m=0.5$ the gradient of the solution develops a singularity near the boundaries $X=-1$ and $X=1$; with $m=1$ the gradient of the solution approaches a non zero constant value near the boundaries $X=-1$ and $X=1$; with $m=2$ the gradient of the solution vanishes as $X$ approaches the edges of the domain. This suggests that $m=1$ is a good choice.

\subsection{Application to volcano ground deformation}

Thanks to the flexibility of the method in dealing with complex geometry, this numerical approach could efficiently afford the modeling of volcano ground deformation caused by pressure changes inside a magma chamber, namely a reservoir of molten rock, where 


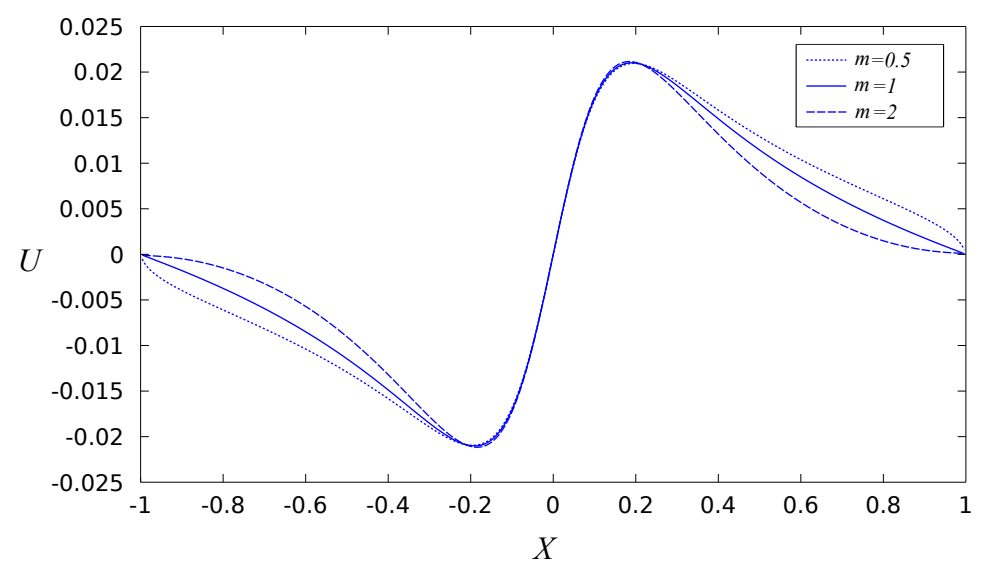

Figure 13: Example 4.2. Numerical solution of the horizontal displacement $U$ for a spherical pressure source on the bounded domain $\Omega^{b}$ along the ground surface $\Gamma_{s}^{b}$ computed with $m=0.5, m=1$ and $m=2$.

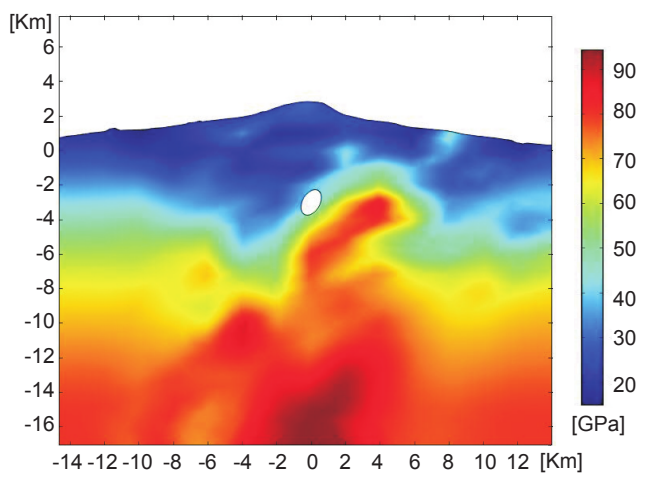

Figure 14: Example 4.3. Real profile of Etna volcano. The color scale refers to the Young modulus $E$.

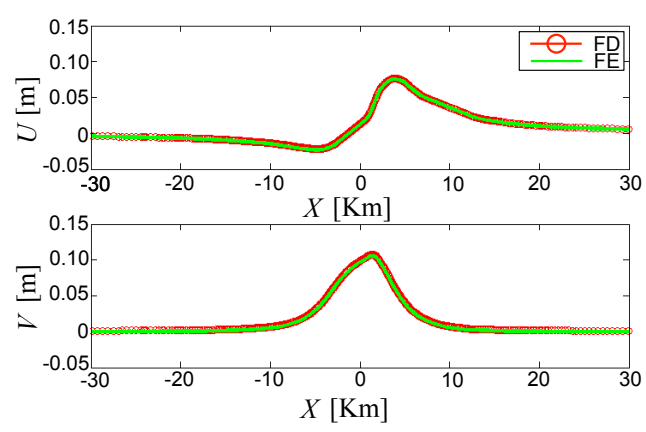

Figure 15: Example 4.3. Comparison between the numerical results for $u$ (top) and $v$ (bottom) obtained by the scheme proposed in this paper (FD) with $N=500$ and the FE method.

magma rising from depths accumulates before being erupted. The volume and pressure state of the magma chamber affect the onset and the size of eruptions and, therefore, it is important to determine the location and geometry of the chamber beneath an active volcano. The pressurization of the magma chamber, produced by a variety of processes such as volatile exsolution and magma recharge, engenders deformation in the surrounding rocks displacing the volcano edifice. The magma chamber pressurization is simulated as a source of deformation $\Omega_{p}$, in which a pressure change acts along its boundary $\Gamma_{p}$ giving rise to deformation. Volcano edifices are complex structures, in which the real topographic profile and medium heterogeneity cannot be disregarded when estimating 
surface displacements. To this aim we build up a simulation where $\Gamma_{S}$ is represented by the real profile of Etna volcano derived using a digital elevation model from the 90 m Shuttle Radar Topography Mission (SRTM) data (Fig. 14). The level-set function of $\Gamma_{s}$ is computed in the whole grid as the minimum (signed) distance between each node of the Cartesian grid and the nodes defining the Etna topography. The boundary $\Gamma_{p}$ of the deformation source is a rotated ellipse simulating a pressurizing magma source. Its level-set is defined as:

$\phi_{p}=\left(\frac{\tilde{x}}{a}\right)^{2}+\left(\frac{\tilde{y}}{b}\right)^{2}-1$, with $\tilde{x}=\left(x-x_{0}\right) \cos \theta-\left(y-y_{0}\right) \sin \theta, \quad \tilde{y}=\left(x-x_{0}\right) \sin \theta+\left(y-y_{0}\right) \cos \theta$,

where $x_{0}=0 \mathrm{~km}, y_{0}=-3 \mathrm{~km}, \theta=\pi / 6, a=0.8 \mathrm{~km}$ and $b=0.5 \mathrm{~km}$. The $2 \mathrm{D}$ plane-strain model of Etna volcano is complemented taking into account the elastic medium properties derived from seismic tomography (Fig. 14). Particularly, the rigidity modulus was estimated by using the following equation [28]:

$$
\mu=\frac{V_{p}^{2} \rho}{3}
$$

where $V_{p}$ is the seismic $P$-wave propagation velocity, and $\rho$ is the density of the medium, which was fixed to a value of $2500 \mathrm{~kg} / \mathrm{m}^{3}$. Instead, the value of Poisson ratio was set to an average value of 0.25 since its variability is limited and does not significantly affect the results [15]. Since neither exact nor analytical solution is available for this test, the numerical results are compared with the solutions obtained from the commercial Finite Element (FE) package COMSOL Multiphysics [12]. The FE computational domain extends $30 \times 30 \mathrm{~km}$ and is discretized non uniformely by a triangular mesh composed of 12677 elements. The mesh is highly refined around the magmatic sources and volcano topography with a resolution of about $0.09 \mathrm{~km}$ and becomes coarser at greater distance reaching a resolution of about $2 \mathrm{~km}$ on the outer most boundaries. The computational domain is surrounded by mapped infinite elements for modeling the unbounded condition $[12,50]$. Second order Lagrange shape functions are used to warrant a third order accuracy [50]. In recent papers the FE solutions have already been validated confirming the goodness of the results $[13-15,18]$. The numerical results match quite well with the FE solution, as shown in Fig. 15, where the displacements computed along $\Gamma_{S}$ are reported. The FE solution is interpolated in the Cartesian grid nodes to compute the relative errors in the $L^{2}$ and $L^{\infty}$ norms for different values of $N$, as shown in Fig. 16.

\section{Conclusion}

A finite-difference ghost-point method for solving the elasto-static equation around a pressure source on an arbitrary unbounded domain has been presented. The proposed strategy adopts the coordinate transformation method to map the original unbounded problem in a bounded one. The benefit of using the coordinate transformation method 

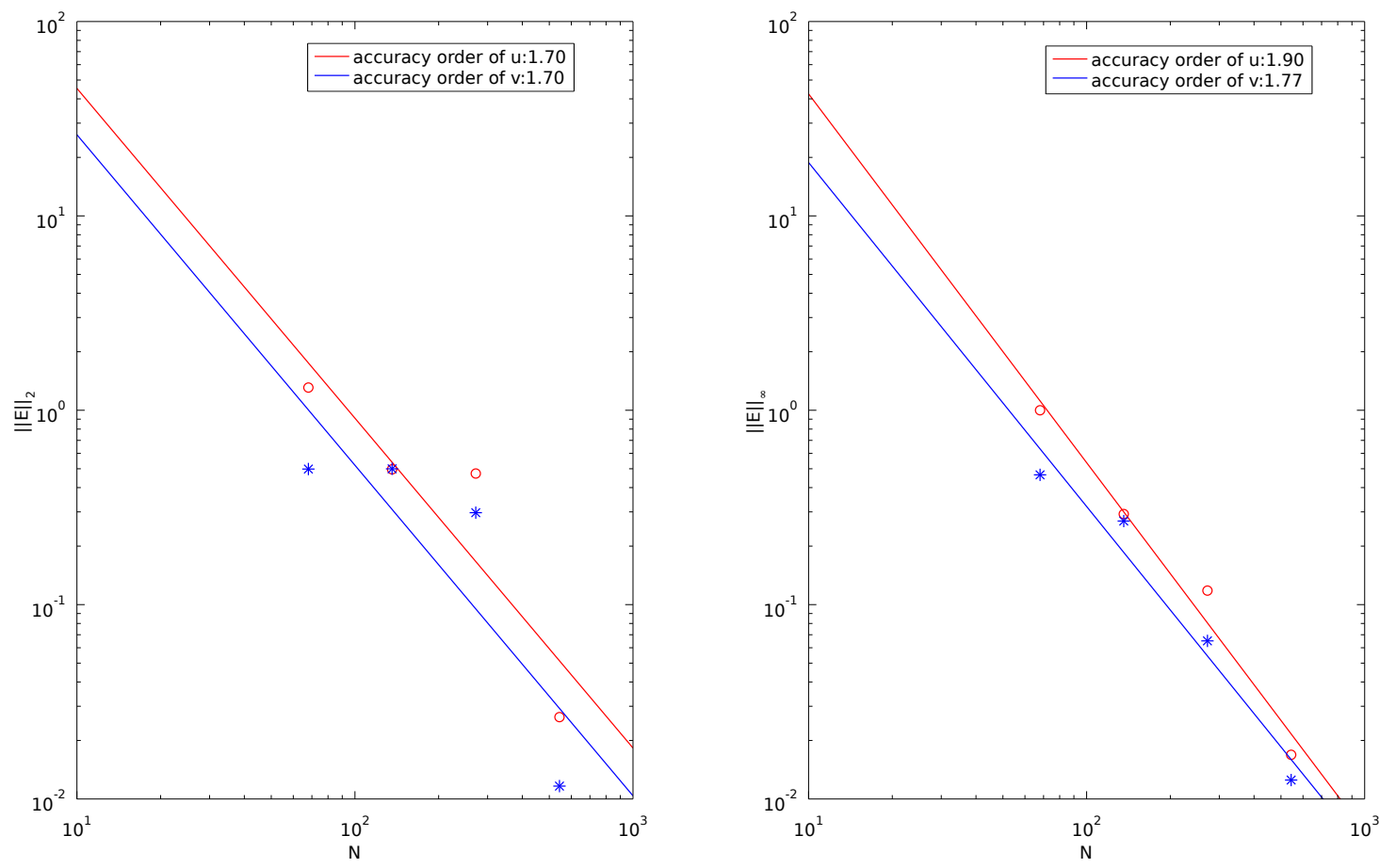

Figure 16: Example 4.3. Bestfit of the $L^{2}$ (left) and $L^{\infty}$ (right) errors of $u$ and $v$. The errors are obtained as the difference from a FE third order accurate solution.

is twofold since it suitably treats unbounded problems and straightforwardly provides a non-uniform grid. Different numerical tests have been performed to validate the method versus analytical and FE results, confirming the second-order accuracy of the solutions. The methodology presents several advantages over the use of FE grids: (i) it is automatically second order accurate with a very compact stencil, (ii) it requires a simpler data structure and (iii) the simple Cartesian grid in the computational domain allows for a very effective geometric multigrid solver. These valuable factors encourage us to extend the method to three dimensional formulation. The practical application of the methodology to volcano ground deformation modeling at Etna shows that the use of the finitedifference ghost-point method could be a useful alternative for simulating the pressurization of magmatic source in the complex structure of a volcanic system and for advancing the model-based assessments of geodetic observations in volcanic areas. Volcano deformation models, simulating magma source pressurization through the solution of the elasto-static equations, provide the linkage between observed displacement at ground surface and the unknown deformation source. Based on a nonlinear inverse analysis, the iterative comparison between the predictions of the deformation model and the observed displacements gives insight into the geometric configuration of the magmatic source. Using the proposed methodology, the non-uniform discretization of the unbounded domain avoids excessive grids far from the source of deformation, yet preserving the versatility 
of a Cartesian grid for the discretization of the elasto-static equations and the boundary conditions while simultaneously accounting for the complex geometry and the internal structure of the volcano. Finally, the embedding of the finite-difference ghost-point method in an iterative inverse scheme, based on the shape evolution of the level-set function, will offer an innovative approach to estimate the nonlinear geometric parameters of the deformation source, reducing computational time and avoiding meshing difficulties related to FEM-based inverse methods.

\section{Acknowledgments}

This work has been supported by the OTRIONS project under the European Territorial Cooperation Programme Greece-Italy 2007-2013, and by PRIN 2009 “Innovative numerical methods for hyperbolic problems with applications to fluid dynamics, kinetic theory and computational biology".

\section{References}

[1] A. B. Alshin. Application of quasi-uniform grids for numerical solution of initial boundary value problems in unbounded domains. In Proceedings of the International Conference on Differential Equations, pages 1024-1026, Hasselt, Belgium, July 1997. World Scientific.

[2] E. Alshina, N. Kalitkin, and S. Panchenko. Numerical solution of boundary value problem in unlimited area. Math. Modelling, 14:10-22, 2002. (in Russian).

[3] J. P. Boyd. The optimization of convergence for Chebyshev polynomial methods in an unbounded domain. Journal of Computational Physics, 45:43-79, 1982.

[4] A. Brandt. Guide to multigrid developments. In W. Hackbusch and U. Trottenberg, editors, Multigrid Methods, Lectures Notes in Mathematics, volume 960, pages 220-312, Berlin, 1982. Springer.

[5] W. L. Briggs, V. E. Henson, and S. F. McCormick. A Multigrid Tutorial. SIAM, 2000.

[6] M. Burger and S. Osher. A survey on level set methods for inverse problems and optimal design. European Journal of Applied Mathematics, 16:263-301, 2005.

[7] J. E. Castillo. Mathematical Aspects of Numerical Grid Generation. Frontiers in Applied Mathematics, 1991.

[8] V. Cayol and H. C. Cornet. Effects of topography on the interpretation of the deformation field of prominent volcanoes - Application to Etna. Geophys. Res. Lett., 25:1979-1982, 1998.

[9] A. Coco and G. Russo. A Ghost-Cell Finite-Difference Multigrid approach for systems of PDE's in arbitrary domains. Submitted.

[10] A. Coco and G. Russo. Finite-Difference Ghost-Point Multigrid Methods on Cartesian Grids for Elliptic Problems in Arbitrary Domains. Journal of Computational Physics, 241:464-501, 2013.

[11] A. Coco, G. Russo, and M. Semplice. Adaptive Mesh Refinement for Hyperbolic Systems based on Third-Order Compact WENO Reconstruction. Submitted.

[12] Comsol AB. Comsol multiphysics 4.3, 2012. Stockholm, Sweden.

[13] G. Currenti, C. Del Negro, A. Di Stefano, and R. Napoli. Numerical simulation of stress induced piezomagnetic fields at Etna volcano. Geophysical Journal International, 179:14691476, 2009. 
[14] G. Currenti, C. Del Negro, and G. Ganci. Modelling of ground deformation and gravity fields using finite element method: an application to Etna volcano. Geophysical Journal International, 169:775-786, 2007.

[15] G. Currenti, C. Del Negro, G. Ganci, and D. Scandura. 3D numerical deformation model of the intrusive event forerunning the 2001 Etna eruption. Physics of the Earth and Planetary Interiors, 168:88-96, 2008.

[16] G. Currenti, C. Del Negro, G. Ganci, and C. A. Williams. Static stress changes induced by the magmatic intrusions during the 2002-2003 Etna eruption. Journal of Geophysical Research, 113, 2008.

[17] F. R. de Hoog and R. Weiss. An approximation theory for boundary value problems on infinite intervals. Computing, 24:227-239, 1980.

[18] C. Del Negro, G. Currenti, and D. Scandura. Temperature-dependent viscoelastic modeling of ground deformation: application to Etna volcano during the 1993-1997 inflation period. Physics of the Earth and Planetary Interiors, 172:299-309, 2009.

[19] A. du Chéné, C. Min, and F. Gibou. Second-Order Accurate Computation of Curvatures in a Level Set Framework Using Novel High Order Reinitialization Schemes. Journal of Scientific Computing archive, 35:114-131, 2008.

[20] R. Fazio and A. Jannelli. Finite difference schemes on quasi-uniform grids for Bvps on infinite intervals. Pre-print available in http://arxiv.org/abs/1211.5427.

[21] Y. Fung. Foundations of solid Mechanics. Prentice-Hall, Englewood Cliffs, 1965.

[22] D. Givoli. Numerical methods for problems in infinite domains. Elsevier, Amsterdam, 1992.

[23] C. E. Grosch and S. A. Orszag. Numerical solution of problems in unbounded regions: coordinate transforms. Journal of Computational Physics, 25:273-295, 1977.

[24] B.-Y. Guo. Jacobi spectral approximations to differential equations on the half line. Journal of Computational Mathematics, 18:95-112, 2000.

[25] D. Han and Z. Huang. A class of artificial boundary conditions for heat equation in unbounded domains. Comp. Math. Appl., 43:889-900, 2002.

[26] M. B. Hend Ben Ameur and, , and B. Hackl. Level set methods for geometric inverse problems in linear elasticity. Inverse Problems, 20:673-696, 2004.

[27] J. Jaeger, N. Cook, and R. Zimmerman. Fundamentals of Rock Mechanics (4th Edition). Blackwell Publishing, Oxford, 2007.

[28] P. Kearey and M. Brooks. An introduction to geophysical exploration. Second edition. Blackwell Scientific Publications, Oxford, 1991.

[29] M. N. Koleva. Numerical Solution of the Heat Equation in Unbounded Domains Using Quasi-uniform Grids. Large-Scale Scientific Computing, Lecture Notes in Computer Science, 3743:509-517, 2006.

[30] M. Lentini and H. B. Keller. Boundary value problems on semi-infinite intervals and their numerical solutions. SIAM J. Numer. Anal., 17:577-604, 1980.

[31] R. Lohner, J. R. Cebral, F. E. Camelli, S. Appanaboyina, J. D. Baum, E. L. Mestreau, and O. A. Soto. Adaptive embedded and immersed unstructured grid techniques. Comput. Methods Appl. Mech. Engrg., 197:217-297, 2008.

[32] M. Maleki, I. Hashim, and S. Abbasbandy. Analysis of IVPs and BVPs on Semi-Infinite Domains via Collocation Methods. Journal of Applied Mathematics, 2012:21 pages, 2012.

[33] P. A. Markowich. A theory for the approximation of solution of boundary value problems on infinite intervals. SIAM J. Math. Anal., 13:484-513, 1982.

[34] P. A. Markowich. Analysis of boundary value problems on infinite intervals. SIAM J. Math. Anal., 14:11-37, 1983. 
[35] T. Masterlark, K. L. Feigl, M. Haney, J. Stone, C. Thurber, and E. Ronchin. Nonlinear estimation of geometric parameters in FEMs of volcano deformation: Integrating tomography models and geodetic data for Okmok volcano, Alaska. J. Geophys. Res., 117:B02407, 2012.

[36] C. Min and F. Gibou. A second order accurate level set method on non-graded adaptive cartesian grids. Journal of Computational Physics, 225:300-321, 2007.

[37] S. Osher and R. Fedkiw. Level Set Methods and Dynamic Implicit Surfaces. Springer-Verlag New York, Applied Mathematical Sciences, 2002.

[38] S. Osher and J. Sethian. Fronts Propagating with Curvature Dependent Speed: Algorithms Based on Hamilton-Jacobi Formulations. Journal of Computational Physics, 79:12-49, 1988.

[39] D. Quang and T. D. Hung. Method of Infinite System of Equations for Problems in Unbounded Domains. Journal of Applied Mathematics, 2012:17 pages, 2012.

[40] G. Russo and P. Smereka. A remark on computing distance functions. Journal of Computational Physics, 163:51-67, 2000.

[41] J. Sethian. Level Set Methods and Fast Marching Methods: Evolving Interfaces in Computational Geometry, Fluid Mechanics, Computer Vision and Materials Science. Cambridge University Press, 1999.

[42] M. Sussman, P. Smereka, and S. Osher. A level set approach for computing solutions to incompressible 2-phase flow. Journal of Computational Physics, 114:146-159, 1994.

[43] E. Trasatti, C. Giunchi, and N. P. Agostinetti. Numerical inversion of deformation caused by pressure sources: application to Mount Etna (Italy). Geophys. J. Int., 172:873-884, 2007.

[44] S. Tsynkov. Numerical solution of problems on unbounded domains. A review. Appl. Numer. Math., 27:465-532, 1989.

[45] U.Trottemberg, C. Oosterlee, and A. Schuller. Multigrid. Academic Press, 2000.

[46] C. Williams and G. Wadge. The effects of topography on magma chamber deformation models: Application to Mt. Etna and radar interferometry. Geophys. Res. Lett., 25:1549-1552, 1998.

[47] C. A. Williams and G. Wadge. An accurate and efficient method for including the effects of topography in three-dimensional elastic models of ground deformation with applications to radar interferometry. J. Geophys. Res., 105:103-120, 2000.

[48] X. Wu and Z. Sun. Convergence of difference scheme for heat equation in unbounded domains using artificial boundary conditions. Appl. Numer. Math., 50:261-277, 2004.

[49] T. Yukutake and H. Tachinaka. Geomagnetic variation associated with stress change within a semi-infinite elastic Earth caused by a cylindrical fource source. Bull. Earthquake Res. Inst., Tokyo, 45:785-798, 1967.

[50] O. Zienkiewicz, C. Emson, and P. Bettess. A Novel Boundary Infinite Element. International Journal for Numerical Methods in Engineering, 19:393-404, 1983. 